\title{
Multi-objective Sustainable Fuzzy Economic Production Quantity (SFEPQ) Model with Demand as Type-2 Fuzzy Number: A Fuzzy Differential Equation Approach
}

\author{
B. K. Debnath*, P. Majumder, U. K. Bera \\ Department of Mathematics, National Institution of Technology, Agartala, Tripura(west), India
}

\begin{abstract}
A sustainable fuzzy economic production quantity (SFEPQ) inventory model is formulated by introducing the concept of fuzzy differential equation (FDE) due to dynamic behavior of the production-demand system. Generalized Hukuhara $(\mathrm{gH})$ differentiability proceedure is applied to solve FDE. Since the demand parameter is taken as trapezoidal type-2 fuzzy number, to get corresponding defuzzified values, first critical value (CV)-based reduction method is applied on demand function to transfer into type-1 fuzzy variable which turns to hexagonal fuzzy number in form. After that $\alpha$-cut of a hexagonal fuzzy number is used to find the upper and lower value of demand. To apply the $\alpha$-cut operation on FDE, we divided the interval $[0,1]$ into two sub-intervals $[0,0.5]$ and $[0.5,1]$ and gH-differentiation is applied on this sub-intervals. The objective of this paper is to maximize the profit and simultaneously minimize the carbon emission cost occurring due to the process of inventory management. Finally, the non-linear objective functions are solved by using of multi-objective genetic algorithm and sensitivity analyses on various parameters are also performed in numerically and graphically.
\end{abstract}

Mathematics Subject Classification (2010). 90B05, 90B30

Keywords. Sustainable economic production quantity model, type-2 fuzzy demand, $\alpha$ cut of hexagonal fuzzy number, fuzzy differential equation, generalized Hukuhara differentiation

\section{Introduction}

Nowadays, many countries have implemented various carbon emission taxes as a part of damage to the environment caused by industry on the inventory process. Therefore, it is a challenge for every manufacturing company or organizations to reduce the carbon emission cost on waste management, excess energy use and obsolescence management by producing sustainable products as well as maintain the profit which motivates the researchers to apply carbon emission factors in their models.

\footnotetext{
${ }^{*}$ Corresponding Author.

Email addresses: deb.bijoy91@yahoo.com (B. K. Debnath), pinki.mjmdr@rediffmail.com (P. Majumder), bera_uttam@yahoo.co.in (U. K. Bera)

Received: 31.07.2017; Accepted: 10.10.2017
} 
Due to the complex environment during the business management, some critical parameters in the inventory problem are always treated as uncertain variables to meet the practical situations. For instance, if one needs to make a decision on inventory management for the next month, the demand and other relevant costs related to inventory are often required to be estimated by professional judgments or probability statistics because of no precise prior information. But, representing demand parameter for an inventory control problem by fuzzy set is considered difficult since it can be determined from many expert's opinion in different ways, and sometimes it is tough to determine the exact membership function. In these cases, each expert's opinion is a membership function of type-1 fuzzy set and thus, this membership function again becomes fuzzy. The final opinion of all experts is expressed by a type-2 fuzzy set (T2 FS).

Also, when the behavior of a dynamical system is not certain, i.e. when the production and demand are fuzzy, the governing differential equation is called fuzzy differential equation (FDE) of instantaneous state of inventory level and the parameters are characterized by a fuzzy number. Hence, we take the demand parameter as trapezoidal type- 2 fuzzy number. In case of a T2 FS, complete defuzzification process consists of two parts-type reduction and defuzzification. Type reduction is a procedure by which a T2 FS is transformed to the corresponding T1 FS, known as type reduced set (TRS). The TRS is then easily defuzzified to a crisp value. Using CV-base reduction method we defuzzified the type-2 fuzzy amount.

The major contribution of this research can be stated as follows:

(i) A profit maximization and carbon emission cost minimization multi-objective partially backlogging fuzzy economic production quantity model is developed where the demand function is taken as trapezoidal type-2 fuzzy variable.

(ii) Fuzzy differential equation proposed by Kandel and Byatt [13] is considered because of the dynamic nature of the system.

(iii) Generalized Hukuhara ( $\mathrm{gH})$ derivative approach proposed by Stefanini and Bede [33] is used to solve the fuzzy differential equation.

(iv) Critical value (CV) -based reduction method is used for trapezoidal type-2 fuzzy variable which become hexagonal fuzzy number in form and $\alpha$-cut of hexagonal fuzzy number is used to get the corresponding crisp value of demand.

(v) Multi-objective genetic algorithm is used to get the corresponding lower and upper bound of profit and carbon emission cost of the non-linear objective function.

\section{Literature survey}

In the literature, it is found that Stock et al. [34] showed that transport and warehouse operations generate large amounts of carbon emission. Hovelaque and Bironneau [11] formulated a carbon constrained integrated economic order quantity (EOQ) model which maximizes a retailer's profit and minimizes carbon emission cost. They investigated the link between inventory policy, total carbon emission and both price and environmental dependent demands. Kazemi et al. [14] formulated an economic order quantity models for items with imperfect quality considering the effect of emission. Battini et al. [3] constructed a new model on sustainable economic order quantity (SEOQ) considering ordering and holding cost of inventory and obsolescence costs and also considered emissions of obsolescence cost for transportation problem. Jonas et al. [12] discussed about the uncertainty present in the greenhouse gas and formulated a fuzzy model in greenhouse gas inventory. Recently, Aljazzar et al. [1] formulated a strategy to reduce carbon emissions from supply chains.

One of the first economic production quantity (EPQ) models with fuzzy parameters was developed by Lee and Yao [17]. In a similar paper, Chang [6] applied the methodology in Lee and Yao [17] and analyzed a condition that the production quantity is a triangular 
fuzzy number (TFN). He deducted that fuzzy and crisp approaches lead to the same result in the investigated model. Another identical research was treated by Lin and Yao [18] who assumed that production quantity is a trapezoidal fuzzy number (TPFN). In this direction, Shekarian et al. [27], [28],[30],[31] formulated different fuzzy EOQ/EPQ models considering different holding costs for imperfect quality items with backorders and rework for a single stage system. Soni et al. [32] formulated a fuzzy inventory model with demand uncertainty and learning in a continuous process. Sadeghi et al. [25] proposed a two-tuned metaheuristics approach for a fuzzy random EPQ problem with shortage and redundancy allocation. The readers could read the extensive survey paper by Shekarian et al. [29] on fuzzy inventory models. All the above investigations assumed the fuzzy parameters/ variables to be of type- 1 fuzzy set (T1 FS). T2 FSs are extensions of T1 FSs was first introduced by Zadeh [38], [39]. The membership grade of a T2 FS is a fuzzy number with a support bounded by the interval $[0,1]$. The logical operations of T2 FS were explored by Mizumoto and Tanaka [22] and Dubois and Prade [8]. Many authors e.g., [19], [26], [35] contributed a large number of theoretical research works on the property of T2 FS and the applications of T2 FS on operations research e.g., [15], [16], [21]. There are several method for type reduction. Qin et al. [23] introduced three kinds of reduction methods called optimistic CV, pessimistic CV and CV reduction methods for T2 FVs based on critical values (CVs) of regular FVs. $\alpha$-cut and the extension principle forms a methodology for extending mathematical concepts from crisp sets to fuzzy sets. These have been applied to many operations and have also been extended to interval valued fuzzy sets. Dubois and Prade [8] has defined fuzzy number as a fuzzy subset of the real line. So far, fuzzy numbers like TFN, TPFN, Hexagonal fuzzy number [24] have been introduced with its membership functions. These numbers have got many applications in practical field and many operations were performed using fuzzy numbers.

The presence of fuzzy demand as well as fuzzy production rate leads to FDE of instantaneous state of inventory level. Till now, FDE is less used to solve fuzzy inventory models though the topics on FDE have been rapidly growing in the recent years. The first impetus on solving FDE was made by Kandel and Byatt [13]. Furthermore, different approaches have been made by several authors to solve FDE [2], [9]. In the FDE, all derivatives are deliberated as either Hukuhara or generalized derivatives. The Hukuhara differentiability [5] has a deficiency that the solution turns fuzzifier as time goes on. Bede [4] exhibited that a large class of Boundary Value Problems (BVPs) has no solution if the Hukuhara derivative is applied. To remove this difficulty, the concept of a generalized derivative was developed and fuzzy differential equations were smeared using this concept. Stefanini and Bede [33] introduced the concept of generalization of the Hukuhara difference for compact convex set, introduced generalized Hukuhara differentiability for fuzzy valued function and they displayed that, this concept of differentiability have relationships with weakly generalized differentiability and strongly generalized differentiability. Villamizar-Roa et al. [36] studied the existence and uniqueness of solution for fuzzy differential equation problems in the setting of a generalized Hukuhara derivative. Guchhait et al. [10] formulated a fuzzy production inventory model using fuzzy differential equation and the corresponding inventory costs and components are calculated using fuzzy Riemann integration. Trade credit financing is one of the central features in supply chain management. In real life situations retailer offers trade credit to his/her customers to boost the demand. This real phenomenon is depicted in our present model. Also, Majumder et al. [20] formulated a fuzzy production inventory model with partial trade credit and solve in fuzzy environment via Generalized Hukuhara derivative approach.

Some papers of the above literature survey and our proposed model are summarized and presented in Table 1.

The rest of the paper is organized as follows: In Section 3, we define all the preliminary concepts relating to fuzzy sets. Section 4, discusses various notations and assumptions. 


\begin{tabular}{|c|c|c|c|c|c|c|c|c|}
\hline Authors & Crisp & Fuzzy & EOQ & EPQ & $\begin{array}{c}\text { Carbon } \\
\text { emission }\end{array}$ & FDE & T2FS & gH-differentiability \\
\hline Hovelaque and Bironneau [11] & $\checkmark$ & & $\checkmark$ & & $\checkmark$ & & & \\
\hline Battini et al. [3] & $\checkmark$ & & $\checkmark$ & & $\checkmark$ & & & \\
\hline Jonas et al. [12] & & $\checkmark$ & & & $\checkmark$ & & & \\
\hline Villamizar-Roa et al. [36] & & $\checkmark$ & & & & $\checkmark$ & & $\checkmark$ \\
\hline Guchhait et al. [10] & & $\checkmark$ & & $\checkmark$ & & $\checkmark$ & & \\
\hline Majumder et al. [20] & & $\checkmark$ & & $\checkmark$ & & $\checkmark$ & & $\checkmark$ \\
\hline Shekarian et al. [30] & & $\checkmark$ & $\checkmark$ & $\checkmark$ & & & & \\
\hline Kazemi et al. [14] & $\checkmark$ & & $\checkmark$ & & $\checkmark$ & & & \\
\hline Proposed Model & & $\checkmark$ & & $\checkmark$ & $\checkmark$ & $\checkmark$ & $\checkmark$ & $\checkmark$ \\
\hline
\end{tabular}

Table 1. Contribution of Different Authors

section 5 is about mathematical formulation of the model. In section 6 , we discuss about the solution procedure for solving multi-objective non-linear problems. In Section 7 real life numerical data and solutions are represented. Discussion on the solution are presented in section 8. Finally brief conclusions and future research work are drawn in section 9 .

\section{Preliminaries}

\subsection{Type-1 Fuzzy set (T1FS) [38]:}

A fuzzy set $\tilde{A}$ is defined by $\tilde{A}=\left\{\left(x, \mu_{\tilde{A}}(x)\right): x \in A, \mu_{\tilde{A}}(x) \in[0,1]\right\}$. In the pair $\left(\mathrm{x}, \mu_{\tilde{A}}(x)\right)$ the first element $\mathrm{x}$ belong to the classical set $\mathrm{A}$, the second element $\mu_{\tilde{A}}(x)$, belong to the interval $[0,1]$, called Membership function.

\subsection{Type-2 Fuzzy Set (T2FS) [38]:}

Type-2 fuzzy set $\tilde{A}$ defined on a universe of discourse $X$, which is denoted as $\tilde{A} \subseteq X$, is a set of pairs $\left\{x, \mu_{\tilde{A}}(x)\right\}$, where $x$ an element of a fuzzy set is, and its grade of membership $\left\{\mu_{\tilde{A}}(x)\right\}$ in the fuzzy set $\tilde{A}$ is a type- 1 fuzzy set defined in the interval $J_{x} \subset[0,1]$, i.e. A T2 FS $\tilde{A}$ is defined as

$$
\tilde{A}=\left\{\left((x, u), \mu_{\tilde{A}}(x, u)\right): \forall x \in X, J_{x} \subset[0,1]\right\},
$$

where $0 \leq \mu_{\tilde{A}}(x, u) \leq 1$ is the type- 2 membership function.

\subsection{Regular fuzzy variable (RFV) [8]:}

For a possibility space $(\varphi, p, P o s)$, a regular fuzzy variable $\tilde{\xi}$ is defined as a measurable map from $\varphi$ to $[0,1]$ in the sense that for every $t \in[0,1]$, one has $\{\gamma \in \varphi \mid \tilde{\xi}(\gamma) \leq t\} \in p$. A discrete RFV is represented as $\tilde{\xi} \sim\left(\begin{array}{c}r_{1}, r_{2} \cdots r_{n} \\ \mu_{1}, \mu_{2} \cdots \mu_{n}\end{array}\right)$ where $r_{i} \in[0,1]$ and $\mu_{i}>0, \forall i$ and $\max _{i}\left(\mu_{i}\right)=1$.

If $\tilde{\xi}=\left(r_{1}, r_{2}, r_{3}, r_{4}\right)$ with $0 \leq r_{1}<r_{2}<r_{3}<r_{4} \leq 1$, then $\tilde{\xi}$ is called a trapezoidal RFV.

Example 3.1. Let us take $\tilde{A}\left\{\left(x, \mu_{\tilde{A}}(x)\right): x \in X\right\}$ where $X=3,6,9$ and the primary memberships of the points $3,6,9$ are given by $J_{3}=0.4,0.8,0.9, J_{6}=0.3,0.7,0.8,0.9$ and $J_{9}=0.2,0.7,1.0$ respectively. Then the secondary grade of the point 3 is

$$
\mu_{\tilde{A}}(3)=\mu_{\tilde{A}}(3, u)=(0.5 / 0.4)+(0.7 / 0.8)+(0.3 / 0.9) \sim\left(\begin{array}{ccc}
0.4 & 0.8 & 0.9 \\
0.5 & 0.7 & 0.3
\end{array}\right)
$$

That means, $\mu_{\tilde{A}}(3,0.4)=0.5, \mu_{\tilde{A}}(3,0.8)=0.7, \mu_{\tilde{A}}(3,0.9)=0.3$.

More specifically $\mu_{\tilde{A}}(3,0.4)=0.5$ means that the membership grade which is named as secondary membership grade that the point 3 has the primary membership 0.4 is 0.5 .

So $\tilde{A}$ considers on the value 3 with membership grade $\left(\begin{array}{ccc}0.4 & 0.8 & 0.9 \\ 0.5 & 0.7 & 0.3\end{array}\right)$, which is a RFV. 


\subsection{Critical values (CVs) for RFVs [23]}

Qin et al. [23] introduced three kinds of critical values (CVs). Let $\tilde{\xi}$ be a RFV. Then,

I. The optimistic $C V$ of $\tilde{\xi}$, denoted by $C V^{*}[\tilde{\xi}]$ is given by,

$$
C V^{*}[\tilde{\xi}]=\sup _{\alpha \in[0,1]}[\alpha \wedge \operatorname{Pos}(\tilde{\xi} \geq \alpha)]
$$

II. The pessimistic $C V$ of $\tilde{\xi}$, denoted by $C V_{*}[\tilde{\xi}]$ is given by,

$$
C V_{*}[\tilde{\xi}]=\sup _{\alpha \in[0,1]}[\alpha \wedge N e c(\tilde{\xi} \geq \alpha)]
$$

III. The $C V$ of $\tilde{\xi}$, denoted by $C V[\tilde{\xi}]$ is given by,

$$
C V[\tilde{\xi}]=\sup _{\alpha \in[0,1]}[\alpha \wedge C r(\tilde{\xi} \geq \alpha)]
$$

Example 3.2. Let $\tilde{\xi}$ be a discrete RFV define as $\xi \sim\left(\begin{array}{cccc}0.3 & 0.5 & 0.8 & 0.9 \\ 0.1 & 0.9 & 0.6 & 0.3\end{array}\right)$

Then we can find out that,

$$
\begin{gathered}
\operatorname{Pos}(\tilde{\xi} \geq \alpha)= \begin{cases}0, & \text { if } \alpha \leq 0.2 \\
0.9, & \text { if } 0.2<\alpha \leq 0.5 \\
0.6, & \text { if } 0.5<\alpha \leq 0.8 \\
0.3, & \text { if } 0.8<\alpha \leq 1.0\end{cases} \\
\operatorname{Nec}(\tilde{\xi} \geq \alpha)= \begin{cases}0.9, & \text { if } \alpha \leq 0.3 \\
0.1, & \text { if } 0.3<\alpha \leq 0.5 \\
0.4, & \text { if } 0.5<\alpha \leq 0.8 \\
0.7, & \text { if } 0.8<\alpha \leq 1.0\end{cases}
\end{gathered}
$$

and

$$
\operatorname{Cr}(\tilde{\xi} \geq \alpha)= \begin{cases}0.9, & \text { if } \alpha \leq 0.3 \\ 0.5, & \text { if } 0.3<\alpha \leq 0.5 \\ 0.5, & \text { if } 0.5<\alpha \leq 0.8 \\ 0.5, & \text { if } 0.8<\alpha \leq 1.0\end{cases}
$$

Then by the definitions of CVs, from (3.2)-(3.4), we have

$$
\begin{aligned}
C V^{*}[\tilde{\xi}] & =\sup _{\alpha \in[0,1]}[\alpha \wedge \operatorname{Pos}(\tilde{\xi} \geq \alpha)] \\
& =\sup _{\alpha \in[0,0.2]}[\alpha \wedge 0] \vee \sup _{\alpha \in(0.2,0.5]}[\alpha \wedge 0.9] \vee \sup _{\alpha \in(0.5,0.8]}[\alpha \wedge 0.6] \vee \sup _{\alpha \in[0.8,1]}(\alpha \wedge 0.3] \\
& =0 \vee 0.5 \vee 0.6 \vee 0.3 \\
& =0.6 \\
C V_{*}[\tilde{\xi}] & =\sup _{\alpha \in[0,1]}[\alpha \wedge N e c(\tilde{\xi} \geq \alpha)] \\
& =\sup _{\alpha \in[0,0.3]}[\alpha \wedge 0.9] \vee \sup _{\alpha \in(0.3,0.5]}[\alpha \wedge 0.1] \vee \sup _{\alpha \in(0.5,0.8]}[\alpha \wedge 0.4] \vee \sup _{\alpha \in(0.8,1]}[\alpha \wedge 0.7] \\
& =0 \vee 0.1 \vee 0.4 \vee 0.7 \\
& =0.7
\end{aligned}
$$

and

$$
\begin{aligned}
C V[\tilde{\xi}] & =\sup _{\alpha \in[0,1]}[\alpha \wedge C r(\tilde{\xi} \geq \alpha)] \\
& =\sup _{\alpha \in[0,0.3]}[\alpha \wedge 0.9] \vee \sup _{\alpha \in(0.3,0.5]}[\alpha \wedge 0.5] \vee \sup _{\alpha \in(0.5,0.8]}[\alpha \wedge 0.5] \vee \sup _{\alpha \in(0.8,1]}[\alpha \wedge 0.5] \\
& =0 \vee 0.5 \vee 0.5 \vee 0.5 \\
& =0.5
\end{aligned}
$$




\subsection{Critical values (CVs) of trapezoidal RFVs [23]}

The following theorems introduced the critical values (CVs) of trapezoidal RFVs.

Theorem 3.3. (Qin et al. [23]) Let $\tilde{\xi}=\left(r_{1}, r_{2}, r_{3}, r_{4}\right)$ be a trapezoidal RFV. Then we have,

(1) The optimistic $C V$ of $\tilde{\xi}$ is $C V^{*}[\tilde{\xi}]=\frac{r_{4}}{\left(1+r_{4}-r_{3}\right)}$.

(2) The pessimistic $C V$ of $\tilde{\xi}$ is $C V_{*}[\tilde{\xi}]=\frac{r_{2}}{\left(1+r_{2}-r_{1}\right)}$.

(3) The $C V$ of $\tilde{\xi}$ is $C V[\tilde{\xi}]= \begin{cases}\frac{2 r_{2}-r_{1}}{1+2\left(r_{2}-r_{1}\right)}, & \text { if } r_{2} \geq \frac{1}{2} \\ \frac{1}{2}, \frac{r_{4}}{1+2\left(r_{4}-r_{3}\right)}, & \text { if } r_{2} \leq \frac{1}{2}<r_{3} \leq \frac{1}{2}\end{cases}$

Example 3.4. Let $\tilde{\xi}=(0.3,0.4,0.8,0.9)$ be a trapezoidal RFV. Then according to the theorem 3.3 we have,

$$
C V^{*}[\tilde{\xi}]=\frac{9}{11}, \quad C V_{*}[\tilde{\xi}]=\frac{4}{11}, \quad C V[\tilde{\xi}]=\frac{1}{2} .
$$

\subsection{Proposed $C V$ based defuzification for trapezoidal type-2 fuzzy vari- able}

According to Chen et al. [7] for a trapezoidal type-2 fuzzy variable $\tilde{\xi}=\left(r_{1}, r_{2}, r_{3}, r_{4} ; \theta_{l}, \theta_{r}\right)$, where $r_{i} \in R, \forall i$ and $\theta_{l}, \theta_{r} \in[0,1]$ are the two parameters that characterize the degree of uncertainty that $\tilde{\xi}$ takes a value say $x$ and the corresponding secondary possibility distribution function $\tilde{\mu}_{\tilde{\xi}}(x)$ is given by,

For any $x \in\left[r_{1}, r_{2}\right]$,

$\tilde{\mu}_{\tilde{\xi}}(x)=\left(\frac{x-r_{1}}{r_{2}-r_{1}}-\theta_{l} \min \left\{\frac{x-r_{1}}{r_{2}-r_{1}}, \frac{r_{2}-x}{r_{2}-r_{1}}\right\}, \frac{x-r_{1}}{r_{2}-r_{1}}, \frac{x-r_{1}}{r_{2}-r_{1}}+\theta_{r} \min \left\{\frac{x-r_{1}}{r_{2}-r_{1}}, \frac{r_{2}-x}{r_{2}-r_{1}}\right\}\right)$

for $x \in\left(r_{2}, r_{3}\right), \quad \tilde{\mu}_{\tilde{\xi}}(x)=\tilde{1}$ and

$\tilde{\mu}_{\tilde{\xi}}(x)=\left(\frac{r_{4}-x}{r_{4}-r_{3}}-\theta_{l} \min \left\{\frac{r_{4}-x}{r_{4}-r_{3}}, \frac{x-r_{3}}{r_{4}-r_{3}}\right\}, \frac{r_{4}-x}{r_{4}-r_{3}}, \frac{r_{4}-x}{r_{4}-r_{3}}+\theta_{r} \min \left\{\frac{r_{4}-x}{r_{4}-r_{3}}, \frac{x-r_{3}}{r_{4}-r_{3}}\right\}\right)$

For any $x \in\left[r_{3}, r_{4}\right]$.

Theorem 3.5. Let $\tilde{\xi}=\left(r_{1}, r_{2}, r_{3}, r_{4} ; \theta_{l}, \theta_{r}\right)$ be a type -2 trapezoidal fuzzy variable. Then we have,

I. Using the optimistic $C V$ reduction method, the reduction $\xi_{1}$ of $\tilde{\xi}$ has the following possibility distribution,

$$
\mu_{\xi_{1}}(x)= \begin{cases}\frac{\left(1+\theta_{r}\right)\left(x-r_{1}\right)}{r_{2}-r_{1}+\theta_{r}\left(x-r_{1}\right)}, & \text { if } x \in\left[r_{1}, \frac{r_{1}+r_{2}}{2}\right] \\ \frac{\left(1-\theta_{r}\right) x-r_{1}+\theta_{r} r_{2}}{r_{2}-r_{1}+\theta_{r}\left(r_{2}-x\right)}, & \text { if } x \in\left(\frac{r_{1}+r_{2}}{2}, r_{2}\right] \\ \tilde{1}, & \text { if } x \in\left(r_{2}, r_{3}\right) \\ \frac{\left(1+\theta_{r}\right)\left(r_{4}-x\right)}{r_{4}-r_{3}+\theta_{r}\left(r_{4}-x\right)}, & \text { if } x \in\left(r_{3}, \frac{r_{3}+r_{4}}{2}\right] \\ \frac{\left(-1+\theta_{r}\right) x-\theta_{r} r_{3}+r_{4}}{r_{4}-r_{3}+\theta_{r}\left(x-r_{4}\right)}, & \text { if } x \in\left(\frac{r_{3}+r_{4}}{2}, r_{4}\right] .\end{cases}
$$


II. Using the pessimistic $C V$ reduction method, the reduction $\xi_{2}$ of $\tilde{\xi}$ has the following possibility distribution,

$$
\mu_{\xi_{2}}(x)= \begin{cases}\frac{\left(x-r_{1}\right)}{r_{2}-r_{1}+\theta_{l}\left(x-r_{1}\right)}, & \text { if } x \in\left[r_{1}, \frac{r_{1}+r_{2}}{2}\right] \\ \frac{\left(x-r_{1}\right)}{r_{2}-r_{1}+\theta_{l}\left(r_{1}-x\right)}, & \text { if } x \in\left(\frac{r_{1}+r_{2}}{2}, r_{2}\right] \\ \tilde{1}, & \text { if } x \in\left(r_{2}, r_{3}\right) \\ \frac{\left(r_{4}-x\right)}{r_{4}-r_{3}+\theta_{l}\left(x-r_{3}\right)}, & \text { if } x \in\left(r_{3}, \frac{r_{3}+r_{4}}{2}\right] \\ \frac{\left(r_{4}-x\right)}{r_{4}-r_{3}+\theta_{l}\left(r_{4}-x\right)}, & \text { if } x \in\left(\frac{r_{3}+r_{4}}{2}, r_{4}\right] .\end{cases}
$$

III. Using the $C V$ reduction method, the reduction $\xi_{3}$ of $\tilde{\xi}$ has the following possibility distribution,

$$
\mu_{\xi_{3}}(x)= \begin{cases}\frac{\left(1+\theta_{r}\right)\left(x-r_{1}\right)}{r_{2}-r_{1}+2 \theta_{r}\left(x-r_{1}\right)}, & \text { if } x \in\left[r_{1}, \frac{r_{1}+r_{2}}{2}\right] \\ \frac{\left(1-\theta_{l}\right) x+\theta_{l} r_{2}-r_{1}}{r_{2}-r_{1}+2 \theta_{l}\left(r_{2}-x\right)}, & \text { if } x \in\left(\frac{r_{1}+r_{2}}{2}, r_{2}\right] \\ \tilde{1}, & \text { if } x \in\left(r_{2}, r_{3}\right) \\ \frac{\left(-1+\theta_{l}\right) x-\theta_{l} r_{3}+r_{4}}{r_{4}-r_{3}+2 \theta_{l}\left(x-r_{3}\right)}, & \text { if } x \in\left(r_{3}, \frac{r_{3}+r_{4}}{2}\right] \\ \frac{\left(1+\theta_{r}\right)\left(r_{4}-x\right)}{r_{4}-r_{3}+2 \theta_{r}\left(r_{4}-x\right)}, & \text { if } x \in\left(\frac{r_{3}+r_{4}}{2}, r_{4}\right] .\end{cases}
$$

From the above theorem we can conclude that when the reduction of trapezoidal type-2 fuzzy variable is made by optimistic CV-reduction method, the possibility distribution function is constructed by use of $\theta_{r}$ and similarly for construction of pessimistic CVreduction of trapezoidal type-2 fuzzy variable $\theta_{l}$ is use. But in case of CV-reduction of trapezoidal type-2 fuzzy variable both $\theta_{l}$ and $\theta_{r}$ are used. Thus, CV-reduction gives more accurate normal value rather than optimistic or pessimistic CV-reduction. Therefore, we take CV-reduction method for our future calculation.

\subsection{Fuzzy number [37]}

A fuzzy number is an extension of a regular number in the sense that it does not refer to one single value but rather than a connected set of possible values. Thus, a fuzzy number is a fuzzy set like $u: R \longrightarrow I=[0,1]$ which satisfies

(1) $u$ is upper semi-continuous.

(2) $u(x)=0$ outside the interval $[c, d]$.

(3) There are real numbers a,b such $c \leq a \leq b \leq d$ and

(i) $u(x)$ is monotonic increasing on $[c, a]$,

(ii) $u(x)$ is monotonic decreasing on $[b, d]$,

(iii) $u(x)=1, a \leq x \leq b$.

\subsection{Hexagonal fuzzy number [24]}

A fuzzy number $\tilde{A}_{h}$ is called a hexagonal fuzzy number, denoted by $\tilde{A}_{h}=\left(a_{1}, a_{2}, a_{3}, a_{4}, a_{5}, a_{6}\right)$ where $a_{1}, a_{2}, a_{3}, a_{4}, a_{5}, a_{6}$ are real numbers and its membership function $\mu_{\tilde{A}_{h}}(x)$ is given below 


$$
\mu_{\tilde{A}_{h}}(x)= \begin{cases}0, & \text { if } x<a_{1} \\ \frac{1}{2}\left[\frac{x-a_{1}}{a_{2}-a_{1}}\right], & \text { if } a_{1} \leq x \leq a_{2} \\ \frac{1}{2}+\frac{1}{2}\left[\frac{x-a_{2}}{a_{3}-a_{2}}\right], & \text { if } a_{2} \leq x \leq a_{3} \\ 1, & \text { if } a_{3} \leq x \leq a_{4} \\ 1-\frac{1}{2}\left[\frac{x-a_{4}}{a_{5}-a_{4}}\right], & \text { if } a_{4} \leq x \leq a_{5} \\ \frac{1}{2}\left[\frac{a_{5}-x}{a_{5}-a_{4}}\right], & \text { if } a_{5} \leq x \leq a_{6} \\ 0, & \text { if } x>a_{6}\end{cases}
$$

Remark: In other words, a hexagonal fuzzy number $\tilde{A}_{h}$ is an ordered quadruple $\left(P_{1}(u), Q_{1}(v), Q_{2}(v), P_{2}(u)\right)$, for $u \in[0,0.5)$ and $v \in[0.5,1)$ where,

(1) $P_{1}(u)=\frac{1}{2}\left[\frac{u-a_{1}}{a_{2}-a_{1}}\right]$ is a bounded continuous non-decreasing function over $[0,0.5)$.

(2) $Q_{1}(v)=\frac{1}{2}+\frac{1}{2}\left[\frac{v-a_{2}}{a_{3}-a_{2}}\right]$ is a bounded continuous non-decreasing function over $[0.5,1]$.

(3) $Q_{2}(v)=1-\frac{1}{2}\left[\frac{v-a_{4}}{a_{5}-a_{4}}\right]$ is a bounded continuous non-decreasing function over $[0.5,1]$.

(4) $P_{2}(u)=\frac{1}{2}\left[\frac{a_{6}-u}{a_{6}-a_{5}}\right]$ is a bounded continuous non-decreasing function over $[0,0.5)$.

\section{9. $\alpha$-cut of fuzzy set [37]}

The $\alpha$-level set (or interval of confidence at level $\alpha$ or $\alpha$-cut) of the fuzzy set $\tilde{A}$ of $\mathrm{X}$ is a crisp set $A_{\alpha}$ that contains all the elements of $\mathrm{X}$ that have membership values greater than or equal to $\alpha$, i.e. $\tilde{A}=\left\{x: \mu_{\tilde{A}}(x) \geq \alpha, x \in X, \alpha \in[0,1]\right\}$.

In case of hexagonal fuzzy number $\tilde{A}_{h}=\left(a_{1}, a_{2}, a_{3}, a_{4}, a_{5}, a_{6}\right)$, the $\alpha$-cut of $\tilde{A}_{h}$ is defined as

$A_{\alpha}=\left\{x \in X: \mu_{\tilde{A}_{h}}(x) \geq x\right\}$

$= \begin{cases}{\left[P_{1}(\alpha), P_{2}(\alpha)\right]} & \text { for } \alpha \in[0,0.5] \\ {\left[Q_{1}(\alpha), Q_{2}(\alpha)\right]} & \text { for } \alpha \in[0.5,1]\end{cases}$

\subsection{0. $\alpha$-cut operations [37]:}

If we get crisp interval by $\alpha$-cut operations interval $A_{\alpha}$ shall be obtained as follows, for all $\alpha \in[0,1]$

Consider, $Q_{1}(x)=\alpha$

$\frac{1}{2}+\frac{1}{2}\left[\frac{x-a_{2}}{a_{3}-a_{2}}\right]=\alpha$

Hence, $Q_{1}(\alpha)=2 \alpha\left(a_{3}-a_{2}\right)+2 a_{2}-a_{3}$

Similarly, $Q_{2}(x)=\alpha, Q_{2}(\alpha)=2 a_{5}-a_{4}-2 \alpha\left(a_{5}-a_{4}\right), P_{1}(\alpha)=2 \alpha\left(a_{2}-a_{1}\right)+a_{1}$

$P_{2}(\alpha)=a_{6}-2 \alpha\left(a_{6}-a_{5}\right)$

Hence,

$$
A_{\alpha}= \begin{cases}{\left[2 \alpha\left(a_{2}-a_{1}\right)+a_{1}, a_{6}-2 \alpha\left(a_{6}-a_{5}\right)\right]} & \text { for } \alpha \in[0,0.5] \\ {\left[2 \alpha\left(a_{3}-a_{2}\right)+2 a_{2}-a_{3}, 2 a_{5}-a_{4}-2 \alpha\left(a_{5}-a_{4}\right)\right]} & \text { for } \alpha \in[0.5,1]\end{cases}
$$

\subsection{1. $\alpha$-cut operation on reduction of a trapezoidal type-2 fuzzy variable}

A trapezoidal type-2 fuzzy fuzzy variable is defined as $\tilde{\xi}=\left(r_{1}, r_{2}, r_{3}, r_{4} ; \theta_{l}, \theta_{r}\right)$, then we have already discussed about reduction method of trapezoidal type-2 fuzzy variable by optimistic $C V$, pessimistic $C V$ and $C V$ reduction.

Now according to the definition of $\alpha$-cut [37], we have the following $\alpha$-cuts of the reductions of $\tilde{\xi}$ 
I. Using the optimistic $C V$ reduction method,

$$
\begin{aligned}
& \xi_{1 L}(\alpha)= \begin{cases}\frac{\left(1+\theta_{r}\right) r_{1}+\left(r_{2}-r_{1}-\theta_{r} r_{1}\right) \alpha}{\left(1+\theta_{r}\right)-\theta_{r} \alpha}, & \text { if } 0 \leq \alpha \leq 0.5 \\
\frac{\left(r_{1}-\theta_{r} r_{1}\right)+\left(r_{2}-r_{1}-\theta_{r} r_{2}\right) \alpha}{\left(1-\theta_{r}\right)+\theta_{r} \alpha}, & \text { if } 0.5<\alpha \leq 1\end{cases} \\
& \xi_{1 R}(\alpha)= \begin{cases}\frac{\left(r_{4}-\theta_{r} r_{3}\right)-\left(r_{4}-r_{3}-\theta_{r} r_{3}\right) \alpha}{\left(1-\theta_{r}\right)-\theta_{r} \alpha}, & \text { if } 0.5 \leq \alpha \leq 1 \\
\frac{\left(1+\theta_{r}\right) r_{4}-\left(r_{4}-r_{3}+\theta_{r} r_{4}\right) \alpha}{\left(1+\theta_{r}\right)-\theta_{r} \alpha}, & \text { if } 0 \leq \alpha \leq 0.5\end{cases}
\end{aligned}
$$

II. Using the pessimistic $C V$ reduction method,

$$
\begin{aligned}
& \xi_{2 L}(\alpha)= \begin{cases}\frac{r_{1}+\left(r_{2}-r_{1}-\theta_{r} r_{1}\right) \alpha}{1-\theta_{l} \alpha}, & \text { if } 0 \leq \alpha \leq 0.5 \\
\frac{r_{1}+\left(r_{2}-r_{1}+\theta_{r} r_{2}\right) \alpha}{1+\theta_{l} \alpha}, & \text { if } 0.5<\alpha \leq 1\end{cases} \\
& \xi_{2 R}(\alpha)= \begin{cases}\frac{r_{4}-\left(r_{4}-r_{3}-\theta_{l} r_{2}\right) \alpha}{1+\theta_{l} \alpha}, & \text { if } 0.5 \leq \alpha \leq 1 \\
\frac{r_{4}-\left(r_{4}-r_{3}+\theta_{l} r_{4}\right) \alpha}{1-\theta_{l} \alpha}, & \text { if } 0 \leq \alpha<0.5\end{cases}
\end{aligned}
$$

III. Using the $C V$ reduction method,

$$
\begin{aligned}
& \xi_{3 L}(\alpha)= \begin{cases}\frac{\left(1+\theta_{r}\right) r_{1}+\left(r_{2}-r_{1}-2 \theta_{r} r_{1}\right) \alpha}{\left(1+\theta_{r}\right)-2 \theta_{r} \alpha}, & \text { if } 0 \leq \alpha \leq 0.5 \\
\frac{\left(r_{1}-\theta_{l} r_{2}+\left(r_{2}-r_{1}-\theta_{l} r_{2}\right) \alpha\right.}{\left(1-\theta_{l}\right)+2 \theta_{l} \alpha}, & \text { if } 0.5<\alpha \leq 1\end{cases} \\
& \xi_{3 R}(\alpha)= \begin{cases}\frac{\left(r_{4}-\theta_{l} r_{3}\right)+\left(r_{4}-r_{3}-2 \theta_{l} r_{3}\right) \alpha}{\left(1-\theta_{l}\right)+2 \theta_{l} \alpha}, & \text { if } 0.5 \leq \alpha \leq 1 \\
\frac{\left(1+\theta_{r}\right) r_{4}-\left(r_{4}-r_{3}+2 \theta_{r} r_{4}\right) \alpha}{\left(1+\theta_{r}\right)-2 \theta_{r} \alpha}, & \text { if } 0 \leq \alpha<0.5\end{cases}
\end{aligned}
$$

By CV reduction method, membership function of type two fuzzy variable $\tilde{\xi}=\left(r_{1}, r_{2}, r_{3}, r_{4} ; \theta_{l}, \theta_{r}\right)$ reduces to membership function of type one variable which is just like a hexagonal fuzzy number. Therefore $\alpha$-cut of $\tilde{\xi}$ is

$[\tilde{\xi}]_{\alpha}= \begin{cases}{\left[P_{1}(\alpha), P_{2}(\alpha)\right]} & \text { for } \alpha \in[0,0.5] \\ {\left[Q_{1}(\alpha), Q_{2}(\alpha)\right]} & \text { for } \alpha \in[0.5,1]\end{cases}$

where,

$$
\begin{array}{ll}
P_{1}(\alpha)=\frac{\left(1+\theta_{r}\right) r_{1}+\left(r_{2}-r_{1}-2 \theta_{r} r_{1}\right) \alpha}{\left(1+\theta_{r}\right)-2 \theta_{r} \alpha}, & P_{2}(\alpha)=\frac{\left(1+\theta_{r}\right) r_{4}-\left(r_{4}-r_{3}+2 \theta_{r} r_{4}\right) \alpha}{\left(1+\theta_{r}\right)-2 \theta_{r} \alpha} \\
Q_{1}(\alpha)=\frac{\left(r_{1}-\theta_{l} r_{2}\right)+\left(r_{2}-r_{1}-\theta_{l} r_{2}\right) \alpha}{\left(1-\theta_{l}\right)+2 \theta_{l} \alpha}, & Q_{2}(\alpha)=\frac{\left(r_{4}-\theta_{l} r_{3}\right)+\left(r_{4}-r_{3}-2 \theta_{l} r_{3}\right) \alpha}{\left(1-\theta_{l}\right)+2 \theta_{l} \alpha}
\end{array}
$$

\section{Notations and assumptions}

To formulate the mathematical model for the proposed inventory system, the following notations and assumptions are made.

\subsection{Notation}

\section{Decision Variables:}

$M$ : Permissible delay period (time) for the retailer offered by the wholesaler, $M>0$.

$N$ : Permissible delay period (time) for the customer offered by the retailer, $0<N<M$.

$T$ : Business period i.e., time period for the cycle of the system, $T>0$.

\section{Parameters:}

$C_{3}=$ Fixed set-up cost $(\$ /$ set up).

$C_{s}=$ Unit selling price $(\$ /$ unit).

$C_{s}^{\prime}=$ Scrap price per unit $(\$ /$ unit $)$.

$p=$ Unit production cost $(\$ /$ unit).

$\alpha^{\prime}=$ Obsolescence rate of inventory (percent).

$b^{\prime}=$ Required space for each unit of product $\left(\mathrm{m}^{3} / \mathrm{unit}\right)$.

$a^{\prime}=$ The weight of the obsolescence product stored in the warehouse (ton $\left./ \mathrm{m}^{3}\right)$.

$C_{b}=$ Backordering cost per unit quantity per unit of time (\$/unit/time). 
$C_{m c}=$ The emission cost of carbon for manufacturing each unit $\left(\$ / m^{3}\right)$.

$C_{o c}=$ Average disposal,waste collection and emission cost for inventory obsolescence $(\$$ $\left./ m^{3}\right)$.

$C_{h}=$ Holding cost per unit item per unit time ( $\$ /$ unit).

$C_{h c}=$ Average emission cost of carbon for holding inventory $\left(\$ / \mathrm{m}^{3}\right)$.

$i_{p}=$ Rate of interest per year per unit to be paid for the unsold inventory after the credit period $M, i_{p}>0$ (\$/year/unit).

$i_{e}=$ Rate of interest per year per unit to be earned from the revenue sold till the time horizon $\mathrm{T}\left(\$ /\right.$ year/unit) $\left(i_{e}<i_{p}\right)$.

\subsection{Assumptions}

The model is developed with the following assumptions.

(1) Production system involves only one non-deteriorating item.

(2) Shortages are allowed with partial backordering.

(3) D, rate of demand depends on the production price and stock i.e., $D(p, q)= \begin{cases}p^{-\epsilon}(\tilde{a}+b q) & \text { if } q>0 \\ \tilde{a} p^{-\epsilon} & \text { if } q \leq 0\end{cases}$

Where $\tilde{a}=\left(r_{1}, r_{2}, r_{3}, r_{4}, \theta_{l}, \theta_{r}\right)$ is a trapezoidal type two fuzzy number and $0<$ $\epsilon<1, r_{1}, r_{2}, r_{3}, r_{4}>0,0<\theta_{l}, \theta_{r}<1$ and $\mathrm{b}$ is any positive real number.

(4) $\mathrm{K}$, rate of production is linearly demand dependent i.e., of the form $K=\mu D$ and $\mu>1$

(5) Rate of earning interest by the retailer is lesser than the rate of interest paid to the wholesaler by the retailer, i.e. $i_{e}<i_{p}$.

(6) Credit period offered by the retailer is smaller than that offered by the wholesaler, i.e. $N<M$.

(7) Customer maintains the trade credit policy offered by the retailer.

\section{Mathematical formulation of the model}

Let the retailer fails to fulfill the demand initially and hence shortages arise from time $t=0$ to the time $t=t_{1}$ and maximum shortage level $Q_{s}$ occur at $t=t_{1}$. After that production process starts to backlog the shortage quantities with partial backordering process and at time $t=t_{2}$ the shortage level reaches to zero. In the mean time inventory accumulates upto time $t=t_{3}$ of amount $Q_{m}$. At that time production process being stop and the accumulated inventory declines to meet up the customers demand and reaches to zero at time $T$.

The governing differential equations of the stock level at any instant $t$ for this model is given by

$$
\frac{d q}{d t}= \begin{cases}-D & 0 \leq t \leq t_{1} \\ K-D & t_{1} \leq t \leq t_{2} \\ K-D & t_{2} \leq t \leq t_{3} \\ -D & t_{3} \leq t \leq T\end{cases}
$$

With the boundary conditions, $q(0)=q\left(t_{2}\right)=q(T)=0$

Bede and Gal [4] applied fuzzy number valued function in fuzzy differential equation and hence, the above equation can be rewritten in fuzzy form

$$
\frac{d \tilde{q}}{d t}= \begin{cases}-\tilde{a} \odot p^{-\epsilon} & 0 \leq t \leq t_{1} \\ (\mu-1) \odot \tilde{a} \odot p^{-\epsilon} & t_{1} \leq t \leq t_{2} \\ (\mu-1) \odot p^{-\epsilon} \odot(\tilde{a}+b \odot \tilde{q}) & t_{2} \leq t \leq t_{3} \\ -p^{-\epsilon} \odot(\tilde{a}+b \odot \tilde{q}) & t_{3} \leq t \leq T\end{cases}
$$

For using CV based reduction method for trapezoidal type-2 fuzzy number, we divided the interval for $\alpha \in[0,1]$ into two sub-intervals like $\alpha \in[0,0.5]$ and $\alpha \in[0.5,1]$ as discussed 
in preliminaries. Also Stefanini and Bede [33] shows that any general interval differential equation can be formulated via generalized Hukuhara $(\mathrm{gH})$ derivative in terms of system of ordinary differential equations.

Now, the Generalized Hukuhara derivative [33] of a fuzzy valued function $f:[a, b] \longmapsto R$ at $t_{0}$ is defined as

$$
f^{\prime}\left(t_{0}\right)=\lim _{h \rightarrow 0} \frac{f\left(t_{0}+h\right) \Theta_{g H} f\left(t_{0}\right)}{h}
$$

In parametric form we say that $\mathrm{f}(\mathrm{t})$ is $\mathrm{gH}$-(i) differentiable at $t_{0}$ if

$$
\left[f^{\prime}\left(t_{0}\right)\right]_{\alpha}=\left[f_{L}^{\prime}\left(t_{0}, \alpha\right), f_{R}^{\prime}\left(t_{0}, \alpha\right)\right]
$$

Also, $\mathrm{f}(\mathrm{t})$ is $\mathrm{gH}$-(ii) differentiable at $t_{0}$ if

$$
\left[f^{\prime}\left(t_{0}\right)\right]_{\alpha}=\left[f_{R}^{\prime}\left(t_{0}, \alpha\right), f_{L}^{\prime}\left(t_{0}, \alpha\right)\right]
$$

Depending upon the value of $\alpha$ two possibility aries

(i) $\alpha \in[0,0.5]$

(ii) $\alpha \in[0.5,1]$

If $\alpha \in[\mathbf{0}, \mathbf{0 . 5}]$, then the above equation takes the form

$$
\left[\frac{d q_{L}}{d t}, \frac{d q_{R}}{d t}\right]= \begin{cases}-\left[a_{L}, a_{R}\right] \odot p^{-\epsilon} & 0 \leq t \leq t_{1} \\ (\mu-1) \odot\left[a_{L}, a_{R}\right] \odot p^{-\epsilon} & t_{1} \leq t \leq t_{2} \\ (\mu-1) \odot p^{-\epsilon}\left(\left[a_{L}, a_{R}\right]+b \odot\left[q_{L}, q_{R}\right]\right) & t_{2} \leq t \leq t_{3} \\ -p^{-\epsilon}\left(\left[a_{L}, a_{R}\right]+b \odot\left[q_{L}, q_{R}\right]\right) & t_{3} \leq t \leq T\end{cases}
$$

Therefore two cases arise

Case 1: gH-(i) differentiable

Case 2: gH-(ii) differentiable

Case-1: Therefore, solving the above system via gH-(i) differentiable is equivalent to solve the corresponding simultaneous system (see [33])

$$
\begin{aligned}
& \frac{d q_{L}}{d t}= \begin{cases}-a_{R} p^{-\epsilon} & 0 \leq t \leq t_{1} \\
(\mu-1) a_{L} p^{-\epsilon} & t_{1} \leq t \leq t_{2} \\
(\mu-1) p^{-\epsilon}\left(a_{L}+b q_{L}\right) & t_{2} \leq t \leq t_{3} \\
-p^{-\epsilon}\left(a_{R}+b q_{R}\right) & t_{3} \leq t \leq T\end{cases} \\
& \frac{d q_{R}}{d t}= \begin{cases}-a_{L} p^{-\epsilon} & 0 \leq t \leq t_{1} \\
(\mu-1) a_{R} p^{-\epsilon} & t_{1} \leq t \leq t_{2} \\
(\mu-1) p^{-\epsilon}\left(a_{R}+b q_{R}\right) & t_{2} \leq t \leq t_{3} \\
-p^{-\epsilon}\left(a_{L}+b q_{L}\right) & t_{3} \leq t \leq T\end{cases}
\end{aligned}
$$

After solving using the boundary conditions, $q(0)=q\left(t_{2}\right)=q(T)=0$, we get

$$
\begin{gathered}
q_{L}(t)= \begin{cases}-a_{R} p^{-\epsilon} t & 0 \leq t \leq t_{1} \\
(\mu-1) a_{L} p^{-\epsilon}\left(t-t_{2}\right) & t_{1} \leq t \leq t_{2} \\
\frac{a_{L}}{b}\left[e^{x\left(t-t_{2}\right)}-1\right] & t_{2} \leq t \leq t_{3} \\
-\frac{a_{R}}{b}\left[e^{y(T-t)}-1\right] & t_{3} \leq t \leq T\end{cases} \\
q_{R}(t)= \begin{cases}-a_{L} p^{-\epsilon} t & 0 \leq t \leq t_{1} \\
(\mu-1) a_{R} p^{-\epsilon}\left(t-t_{2}\right) & t_{1} \leq t \leq t_{2} \\
\frac{a_{R}}{b}\left[e^{x\left(t-t_{2}\right)}-1\right] & t_{2} \leq t \leq t_{3} \\
-\frac{a_{L}}{b}\left[e^{y(T-t)}-1\right] & t_{3} \leq t \leq T\end{cases}
\end{gathered}
$$

where, $x=b(\mu-1) p^{-\epsilon}$ and $y=b p^{-\epsilon}$ 
$a_{L}(\alpha)=\frac{\left(1+\theta_{r}\right) r_{1}+\left(r_{2}-r_{1}-2 \theta_{r} r_{1}\right) \alpha}{\left(1+\theta_{r}\right)-2 \theta_{r} \alpha}$

$a_{R}(\alpha)=\frac{\left(1+\theta_{r}\right) r_{4}-\left(r_{4}-r_{3}+2 \theta_{r} r_{4}\right) \alpha}{\left(1+\theta_{r}\right)-2 \theta_{r} \alpha}$

Similarly, to find the lower and upper limit of inventory accumulated upto time $t=t_{3}$ is given by the condition

$\left(Q_{m}\right)_{L}=\frac{a_{L}}{b}\left[e^{x\left(t_{3}-t_{2}\right)}-1\right]=-\frac{a_{R}}{b}\left[e^{y\left(T-t_{3}\right)}-1\right]$

$\left(Q_{m}\right)_{U}=\frac{a_{R}}{b}\left[e^{x\left(t_{3}-t_{2}\right)}-1\right]=-\frac{a_{L}}{b}\left[e^{y\left(T-t_{3}\right)}-1\right]$

Now, the inventory related costs are as follows

Total obsolescence cost of inventory

$T O C_{L}=\alpha^{\prime}\left(C_{s}-C_{s}^{\prime}\right)\left[\int_{t_{2}}^{t_{3}} q_{L}(t) d t+\int_{t_{3}}^{T} q_{L}(t) d t\right]=\alpha^{\prime}\left(C_{s}-C_{s}^{\prime}\right)\left[\frac{a_{L}}{b}\left\{\frac{1}{x}\left(e^{x\left(t_{3}-t_{2}\right)}-1\right)-\left(t_{3}-\right.\right.\right.$

$\left.\left.\left.t_{2}\right)\right\}+\frac{a_{R}}{b}\left\{\frac{1}{y}\left(1-e^{y\left(T-t_{3}\right)}\right)+\left(T-t_{3}\right)\right\}\right]$

$T O C_{R}=\alpha^{\prime}\left(C_{s}-C_{s}^{\prime}\right)\left[\int_{t_{2}}^{t_{3}} q_{R}(t) d t+\int_{t_{3}}^{T} q_{R}(t) d t\right]=\alpha^{\prime}\left(C_{s}-C_{s}^{\prime}\right)\left[\frac{a_{R}}{b}\left\{\frac{1}{x}\left(e^{x\left(t_{3}-t_{2}\right)}-1\right)-\left(t_{3}-\right.\right.\right.$

$\left.\left.\left.t_{2}\right)\right\}+\frac{a_{L}}{b}\left\{\frac{1}{y}\left(1-e^{y\left(T-t_{3}\right)}\right)+\left(T-t_{3}\right)\right\}\right]$

Total cost of emission of inventory obsolescence

$T E O_{L}=\alpha^{\prime} a^{\prime} C_{o c}\left[\int_{t_{2}}^{t_{3}} q_{L}(t) d t+\int_{t_{3}}^{T} q_{L}(t) d t\right]=\alpha^{\prime} a^{\prime} C_{o c}\left[\frac{a_{L}}{b}\left\{\frac{1}{x}\left(e^{x\left(t_{3}-t_{2}\right)}-1\right)-\left(t_{3}-t_{2}\right)\right\}+\right.$ $\left.\frac{a_{R}}{b}\left\{\frac{1}{y}\left(1-e^{y\left(T-t_{3}\right)}\right)+\left(T-t_{3}\right)\right\}\right]$

$T E O_{R}=\alpha^{\prime} a^{\prime} C_{o c}\left[\int_{t_{2}}^{t_{3}} q_{R}(t) d t+\int_{t_{3}}^{T} q_{R}(t) d t\right]=\alpha^{\prime} a^{\prime} C_{o c}\left[\frac{a_{R}}{b}\left\{\frac{1}{x}\left(e^{x\left(t_{3}-t_{2}\right)}-1\right)-\left(t_{3}-t_{2}\right)\right\}+\right.$ $\left.\frac{a_{L}}{b}\left\{\frac{1}{y}\left(1-e^{y\left(T-t_{3}\right)}\right)+\left(T-t_{3}\right)\right\}\right]$

Total backordering cost

$T B C_{L}=C_{b}\left[\int_{0}^{t_{1}} q_{L}(t) d t+\int_{t_{1}}^{t_{2}} q_{L}(t) d t\right]=-\frac{C_{b}}{2}\left[a_{R} p^{-\epsilon} t_{1}^{2}+(\mu-1) a_{L} p^{-\epsilon}\left(t_{1}-t_{2}\right)^{2}\right]$

$T B C_{R}=C_{b}\left[\int_{0}^{t_{1}} q_{R}(t) d t+\int_{t_{1}}^{t_{2}} q_{R}(t) d t\right]=-\frac{C_{b}}{2}\left[a_{L} p^{-\epsilon} t_{1}^{2}+(\mu-1) a_{R} p^{-\epsilon}\left(t_{1}-t_{2}\right)^{2}\right]$

Total goodwill loss for back-order is

$T G C_{L}=C_{g}\left[\int_{0}^{t_{1}} q_{L}(t) d t+\int_{t_{1}}^{t_{2}} q_{L}(t) d t\right]=-\frac{C_{g}}{2}\left[a_{R} p^{-\epsilon} t_{1}^{2}+(\mu-1) a_{L} p^{-\epsilon}\left(t_{1}-t_{2}\right)^{2}\right]$

$T G C_{R}=C_{g}\left[\int_{0}^{t_{1}} q_{L}(t) d t+\int_{t_{1}}^{t_{2}} q_{L}(t) d t\right]=-\frac{C_{g}}{2}\left[a_{L} p^{-\epsilon} t_{1}^{2}+(\mu-1) a_{R} p^{-\epsilon}\left(t_{1}-t_{2}\right)^{2}\right]$

Total holding cost

$T H C_{L}=\int_{t_{2}}^{t_{3}} C_{h} q_{L}(t) d t+\int_{t_{3}}^{T} C_{h} q_{L}(t) d t=C_{h}\left[\frac{a_{L}}{b}\left\{\frac{1}{x}\left(e^{x\left(t_{3}-t_{2}\right)}-1\right)-\left(t_{3}-t_{2}\right)\right\}+\frac{a_{R}}{b}\left\{\frac{1}{y}(1-\right.\right.$ $\left.\left.\left.e^{y\left(T-t_{3}\right)}\right)+\left(T-t_{3}\right)\right\}\right]$

$T H C_{R}=\int_{t_{2}}^{t_{3}} C_{h} q_{R}(t) d t+\int_{t_{3}}^{T} C_{h} q_{R}(t) d t=C_{h}\left[\frac{a_{R}}{b}\left\{\frac{1}{x}\left(e^{x\left(t_{3}-t_{2}\right)}-1\right)-\left(t_{3}-t_{2}\right)\right\}+\frac{a_{L}}{b}\left\{\frac{1}{y}(1-\right.\right.$ $\left.\left.\left.e^{y\left(T-t_{3}\right)}\right)+\left(T-t_{3}\right)\right\}\right]$

Total emission cost of carbon for holding inventory

$T E H_{L}=C_{h c} b^{\prime}\left[\int_{t_{2}}^{t_{3}} q_{L}(t) d t+\int_{t_{3}}^{T} q_{L}(t) d t\right]=C_{h c} b^{\prime}\left[\frac{a_{L}}{b}\left\{\frac{1}{x}\left(e^{x\left(t_{3}-t_{2}\right)}-1\right)-\left(t_{3}-t_{2}\right)\right\}+\frac{a_{R}}{b}\left\{\frac{1}{y}(1-\right.\right.$ $\left.\left.\left.e^{y\left(T-t_{3}\right)}\right)+\left(T-t_{3}\right)\right\}\right]$

$T E H_{R}=C_{h c} b^{\prime}\left[\int_{t_{2}}^{t_{3}} q_{R}(t) d t+\int_{t_{3}}^{T} q_{R}(t) d t\right]=C_{h c} b^{\prime}\left[\frac{a_{R}}{b}\left\{\frac{1}{x}\left(e^{x\left(t_{3}-t_{2}\right)}-1\right)-\left(t_{3}-t_{2}\right)\right\}+\frac{a_{L}}{b}\left\{\frac{1}{y}(1-\right.\right.$ $\left.\left.\left.e^{y\left(T-t_{3}\right)}\right)+\left(T-t_{3}\right)\right\}\right]$

Total set-up cost $=C_{3}$

Total revenue earned

$T R E_{L}=c_{S}\left[\mu a_{L} p^{-\epsilon}\left(t_{2}-t_{1}\right)+\mu \int_{t_{2}}^{t_{3}} p^{-\epsilon}\left(a_{L}+b q_{L}\right) d t\right]=c_{S}\left[\mu a_{L} p^{-\epsilon}\left(t_{2}-t_{1}\right)+\mu p^{-\epsilon} a_{L} e^{-x t_{2}} \int_{t_{2}}^{t_{3}} e^{x t} d t\right]$ $=c_{S} \mu a_{L} p^{-\epsilon}\left[t_{2}-t_{1}+\frac{1}{x}\left(e^{x\left(t_{3}-t_{2}\right)}-1\right)\right]$ 
$T R E_{R}=c_{S}\left[\mu a_{R} p^{-\epsilon}\left(t_{2}-t_{1}\right)+\mu \int_{t_{2}}^{t_{3}} p^{-\epsilon}\left(a_{R}+b q_{R}\right) d t\right]=c_{S}\left[\mu a_{R} p^{-\epsilon}\left(t_{2}-t_{1}\right)+\mu p^{-\epsilon} a_{R} e^{-x t_{2}} \int_{t_{2}}^{t_{3}} e^{x t} d t\right]$ $=c_{S} \mu a_{R} p^{-\epsilon}\left[t_{2}-t_{1}+\frac{1}{x}\left(e^{x\left(t_{3}-t_{2}\right)}-1\right)\right]$

Total Production cost

$T P C_{L}=p\left[\mu a_{L} p^{-\epsilon}\left(t_{2}-t_{1}\right)+\mu \int_{t_{2}}^{t_{3}} p^{-\epsilon}\left(a_{L}+b q_{L}\right) d t\right]=p\left[\mu a_{L} p^{-\epsilon}\left(t_{2}-t_{1}\right)+\mu p^{-\epsilon} a_{L} e^{-x t_{2}} \int_{t_{2}}^{t_{3}} e^{x t} d t\right]$ $=p \mu a_{L} p^{-\epsilon}\left[t_{2}-t_{1}+\frac{1}{x}\left(e^{x\left(t_{3}-t_{2}\right)}-1\right)\right]$

$T P C_{R}=p\left[\mu a_{R} p^{-\epsilon}\left(t_{2}-t_{1}\right)+\mu \int_{t_{2}}^{t_{3}} p^{-\epsilon}\left(a_{R}+b q_{R}\right) d t\right]=p\left[\mu a_{R} p^{-\epsilon}\left(t_{2}-t_{1}\right)+\mu p^{-\epsilon} a_{R} e^{-x t_{2}} \int_{t_{2}}^{t_{3}} e^{x t} d t\right]$ $=p \mu a_{R} p^{-\epsilon}\left[t_{2}-t_{1}+\frac{1}{x}\left(e^{x\left(t_{3}-t_{2}\right)}-1\right)\right]$

The emission cost of carbon for manufacturing total units $T E P_{L}=c_{m c}\left[\mu a_{L} p^{-\epsilon}\left(t_{2}-t_{1}\right)+\mu \int_{t_{2}}^{t_{3}} p^{-\epsilon}\left(a_{L}+b q_{L}\right) d t\right]=c_{m c}\left[\mu a_{L} p^{-\epsilon}\left(t_{2}-t_{1}\right)+\mu p^{-\epsilon} a_{L} e^{-x t_{2}} \int_{t_{2}}^{t_{3}} e^{x t} d t\right]$ $=c_{m c} \mu a_{L} p^{-\epsilon}\left[t_{2}-t_{1}+\frac{1}{x}\left(e^{x\left(t_{3}-t_{2}\right)}-1\right)\right]$

$T E P_{R}=c_{m c}\left[\mu a_{R} p^{-\epsilon}\left(t_{2}-t_{1}\right)+\mu \int_{t_{2}}^{t_{3}} p^{-\epsilon}\left(a_{R}+b q_{R}\right) d t\right]=c_{m c}\left[\mu a_{R} p^{-\epsilon}\left(t_{2}-t_{1}\right)+\mu p^{-\epsilon} a_{R} e^{-x t_{2}} \int_{t_{2}}^{t_{3}} e^{x t} d t\right]$ $=c_{m c} \mu a_{R} p^{-\epsilon}\left[t_{2}-t_{1}+\frac{1}{x}\left(e^{x\left(t_{3}-t_{2}\right)}-1\right)\right]$

Therefore three sub-cases arise depending upon the values of changing time periods.

Sub-case 1.1: $0 \leq t_{1} \leq N \leq M \leq t_{2} \leq t_{3} \leq T$

In this case if the amount is paid within $M$ by the retailer, then there is no interest payable. Otherwise, the retailer will pay for the rest of the inventory. Hence, the total amount of interest paid and interest earned by the retailer is calculated.

Hence, total interest paid

$T I P_{L}=p i_{p}\left[\int_{M}^{t_{2}}\left(1+T-t_{2}\right) q_{L}(t) d t+\int_{t_{2}}^{t_{3}}\left(1+T-t_{3}\right) q_{L}(t) d t+\int_{t_{3}}^{T} q_{L}(t) d t\right]$

$=p i_{p}\left[\frac{1}{2}\left(1+T-t_{2}\right)(\mu-1) a_{L} p^{-\epsilon}\left(t_{2}-M\right)^{2}+\left(1+T-t_{3}\right) \frac{a_{L}}{b}\left\{\frac{1}{x}\left(e^{x\left(t_{3}-t_{2}\right)}-1\right)-\left(t_{3}-t_{2}\right)\right\}+\right.$ $\left.\frac{a_{R}}{b}\left\{\frac{1}{y}\left(1-e^{y\left(T-t_{3}\right)}\right)+\left(T-t_{3}\right)\right\}\right]$

$T I P_{R}=p i_{p}\left[\int_{M}^{t_{2}}\left(1+T-t_{2}\right) q_{R}(t) d t+\int_{t_{2}}^{t_{3}}\left(1+T-t_{3}\right) q_{R}(t) d t+\int_{t_{3}}^{T} q_{R}(t) d t\right]$

$T I P_{R}=C_{p} i_{p}\left[\frac{1}{2}\left(1+T-t_{2}\right)(\mu-1) a_{R} p^{-\epsilon}\left(t_{2}-M\right)^{2}+\left(1+T-t_{3}\right) \frac{a_{R}}{b}\left\{\frac{1}{x}\left(e^{x\left(t_{3}-t_{2}\right)}-1\right)-\left(t_{3}-\right.\right.\right.$ $\left.\left.\left.t_{2}\right)\right\}+\frac{a_{L}}{b}\left\{\frac{1}{y}\left(1-e^{y\left(T-t_{3}\right)}\right)+\left(T-t_{3}\right)\right\}\right]$

And, total interest earned

$T I E_{L}=C_{s} i_{e}\left[(T-N) \int_{t_{1}}^{N} D_{L}(p, q) d t+(1+T-M) \int_{N}^{M} D_{L}(p, q)(M-t) d t+(1+T-\right.$ $\left.\left.t_{2}\right) \int_{M}^{t_{2}} D_{L}(p, q)\left(t_{2}-t\right) d t+\left(1+T-t_{3}\right) \int_{t_{2}}^{t_{3}} D_{L}(p, q)\left(t_{3}-t\right) d t+\int_{t_{3}}^{T} D_{L}(p, q)(T-t) d t\right]$ $=C_{s} i_{e} a_{L} p^{-\epsilon}\left[(T-N)\left(N-t_{1}\right)+\frac{1}{2}(1+T-M)(M-N)^{2}+\frac{1}{2}\left(1+T-t_{2}\right)\left(t_{2}-M\right)^{2}+p^{-\epsilon}\left\{a_{L}-\right.\right.$ $\left.\left.a_{R}\left(e^{y\left(T-t_{3}\right)}-1\right)\right\}\right]$

$T I E_{R}=C_{s} i_{e}\left[(T-N) \int_{t_{1}}^{N} D_{R}(p, q) d t+(1+T-M) \int_{N}^{M} D_{R}(p, q)(M-t) d t+(1+T-\right.$ $\left.\left.t_{2}\right) \int_{M}^{t_{2}} D_{R}(p, q)\left(t_{2}-t\right) d t+\left(1+T-t_{3}\right) \int_{t_{2}}^{t_{3}} D_{R}(p, q)\left(t_{3}-t\right) d t+\int_{t_{3}}^{T} D_{R}(p, q)(T-t) d t\right]$ $=C_{s} i_{e} a_{L} p^{-\epsilon}\left[(T-N)\left(N-t_{1}\right)+\frac{1}{2}(1+T-M)(M-N)^{2}+\frac{1}{2}\left(1+T-t_{2}\right)\left(t_{2}-M\right)^{2}+p^{-\epsilon}\left\{a_{L}-\right.\right.$ $\left.\left.a_{R}\left(e^{y\left(T-t_{3}\right)}-1\right)\right\}\right]$ 
Sub-case 1.2: $0 \leq t_{1} \leq t_{2} \leq N \leq M \leq t_{3} \leq T$

In this case the total amount of interest paid and interest earned by the retailer is calculated.

Hence, total interest paid

$$
\begin{aligned}
& T I P_{L}=p i_{p}\left[\int_{M}^{t_{3}}\left(1+T-t_{3}\right) q_{L}(t) d t+\int_{t_{3}}^{T} q_{L}(t) d t\right] \\
& =p i_{p}\left[\left(1+T-t_{3}\right) \frac{a_{L}}{b}\left\{\frac{1}{x}\left(e^{x\left(t_{3}-M\right)}-1\right)-\left(t_{3}-M\right)\right\}+\frac{a_{R}}{b}\left\{\frac{1}{y}\left(1-e^{y\left(T-t_{3}\right)}\right)+\left(T-t_{3}\right)\right\}\right] \\
& T I P_{L}=p i_{p}\left[\int_{M}^{t_{3}}\left(1+T-t_{3}\right) q_{R}(t) d t+\int_{t_{3}}^{T} q_{R}(t) d t\right] \\
& =p i_{p}\left[\left(1+T-t_{3}\right) \frac{a_{R}}{b}\left\{\frac{1}{x}\left(e^{x\left(t_{3}-M\right)}-1\right)-\left(t_{3}-M\right)\right\}+\frac{a_{L}}{b}\left\{\frac{1}{y}\left(1-e^{y\left(T-t_{3}\right)}\right)+\left(T-t_{3}\right)\right\}\right]
\end{aligned}
$$

Total interest earned

$$
\begin{aligned}
& T I E_{L}=C_{s} i_{e}\left[\left(T-t_{2}\right) \int_{t_{1}}^{t_{2}} D_{L}(p, q) d t+(T-N) \int_{t_{2}}^{N} D_{L}(p, q) d t+\left(1+T-t_{3}\right) \int_{N}^{t_{3}} D_{L}(p, q)\left(t_{3}-\right.\right. \\
& \left.t) d t+(1+T-M) \int_{t_{3}}^{M} D_{L}(p, q)(M-t) d t+\int_{M}^{T} D_{L}(p, q)(T-t) d t\right] \\
& =C_{s} i_{e} a_{L} p^{-\epsilon}\left[\left(T-t_{2}\right)\left(t_{2}-t_{1}\right)+(T-N) \frac{a_{L}}{x}\left(e^{x\left(N-t_{2}\right)}-1\right)+\frac{1}{2}\left(1+T-t_{3}\right)\left(t_{3}-N\right)^{2}+\frac{1}{2}(1+\right. \\
& \left.T-M)\left(M-t_{3}\right)^{2}+p^{-\epsilon}\left\{a_{L}-a_{R}\left(e^{y\left(T-t_{3}\right)}-1\right)\right\}\right] \\
& T I E_{R}=C_{s} i_{e}\left[\left(T-t_{2}\right) \int_{t_{1}}^{t_{2}} D_{R}(p, q) d t+(T-N) \int_{t_{2}}^{N} D_{R}(p, q) d t+\left(1+T-t_{3}\right) \int_{N}^{t_{3}} D_{R}(p, q)\left(t_{3}-\right.\right. \\
& \left.t) d t+(1+T-M) \int_{t_{3}}^{M} D_{R}(p, q)(M-t) d t+\int_{M}^{T} D_{R}(p, q)(T-t) d t\right] \\
& =C_{s} i_{e} a_{R} p^{-\epsilon}\left[\left(T-t_{2}\right)\left(t_{2}-t_{1}\right)+(T-N) \frac{a_{L}}{x}\left(e^{x\left(N-t_{2}\right)}-1\right)+\frac{1}{2}\left(1+T-t_{3}\right)\left(t_{3}-N\right)^{2}+\frac{1}{2}(1+\right. \\
& \left.T-M)\left(M-t_{3}\right)^{2}+p^{-\epsilon}\left\{a_{R}-a_{L}\left(e^{y\left(T-t_{3}\right)}-1\right)\right\}\right]
\end{aligned}
$$

Sub-case 1.3: $0 \leq t_{1} \leq t_{2} \leq N \leq t_{3} \leq T \leq M$

In this case interest payable by the retailer is zero, i.e., $T I P_{L}=T I P_{R}=0$

But, interest earned by the retailer is given by

$T I E_{L}=C_{s} i_{e}\left[\left(M-t_{2}\right) \int_{t_{1}}^{t_{2}} D_{L}(p, q) d t+(M-N) \int_{t_{2}}^{N} D_{L}(p, q) d t+\left(1+M-t_{3}\right) \int_{N}^{t_{3}} D_{L}(p, q)\left(t_{3}-\right.\right.$

$\left.t) d t+(1+M-T) \int_{t_{3}}^{T} D_{L}(p, q)(T-t) d t\right]$

$=C_{s} i_{e} a_{L} p^{-\epsilon}\left[\left(M-t_{2}\right)\left(t_{2}-t_{1}\right)+(M-N)\left(N-t_{1}\right)+\frac{1}{2}\left(1+M-t_{3}\right)\left(t_{3}-N\right)^{2}+\frac{1}{2}(1+M-\right.$

$\left.T)\left(T-t_{3}\right)^{2}+\left\{a_{L}-a_{R}\left(e^{y\left(T-t_{3}\right)}-1\right)\right\}\right]$

$T I E_{R}=C_{s} i_{e}\left[\left(M-t_{2}\right) \int_{t_{1}}^{t_{2}} D_{R}(p, q) d t+(M-N) \int_{t_{2}}^{N} D_{R}(p, q) d t+\left(1+M-t_{3}\right) \int_{N}^{t_{3}} D_{R}(p, q)\left(t_{3}-\right.\right.$

$\left.t) d t+(1+M-T) \int_{t_{3}}^{T} D_{R}(p, q)(T-t) d t\right]$

$=C_{s} i_{e} a_{R} p^{-\epsilon}\left[\left(M-t_{2}\right)\left(t_{2}-t_{1}\right)+(M-N)\left(N-t_{1}\right)+\frac{1}{2}\left(1+M-t_{3}\right)\left(t_{3}-N\right)^{2}+\frac{1}{2}(1+M-\right.$

$\left.T)\left(T-t_{3}\right)^{2}+\left\{a_{R}-a_{L}\left(e^{y\left(T-t_{3}\right)}-1\right)\right\}\right]$

Here, a carbon emissions integrated fuzzy EPQ model with type-2 fuzzy variable is considered, where the objectives are maximizing the profit and minimizing various carbon emission costs associated with inventory management. By using gH-differentiability, we get a range for profit and a range of emission rather than an exact value of profit and emission, which is more realistic in practical value. Hence, the objective functions are defined as follows:

Max Profit $(T P)=\frac{1}{T}\left[T R E+T I E-T P C-T H C-T O C-T I P-T B C-T G C-C_{3}^{3}\right)$

Min Carbon Emission Cost $(T E)=T E O+T E H+T E P$ 
On taking $\alpha$-cut over the total profit and emission per unit time is a interval crisp set and is defined by

$T P=\left[T P_{L}, T P_{R}\right]$ where,

$T P_{L}=\frac{1}{T}\left[T R E_{L}+T I E_{L}-T P C_{L}-T H C_{L}-T O C_{L}-T I P_{L}-T B C_{L}-T G C_{L}-C_{3}\right]$

$T P_{R}=\frac{1}{T}\left[T R E_{R}+T I E_{R}-T P C_{R}-T H C_{R}-T O C_{R}-T I P_{R}-T B C_{R}-T G C_{R}-C\right.$

And, $T E=\left[T E_{L}, T E_{R}\right]$ where,

$T E_{L}=T E O_{L}+T E H_{L}+T E P_{L}$

$T E_{R}=T E O_{R}+T E H_{R}+T E P_{R}$, when $0 \leq \alpha \leq 0.5$

Case-2: If $\alpha \in[0,0.5]$, then on taking the $\alpha$-cut of fuzzy differential equation reduces to interval fuzzy differential equation via gH-(ii) differentiability

$$
\left[\frac{d q_{L}}{d t}, \frac{d q_{R}}{d t}\right]= \begin{cases}-\left[a_{L}, a_{R}\right] \odot p^{-\epsilon} & 0 \leq t \leq t_{1} \\ (\mu-1) \odot\left[a_{L}, a_{R}\right] \odot p^{-\epsilon} & t_{1} \leq t \leq t_{2} \\ (\mu-1) \odot p^{-\epsilon}\left(\left[a_{L}, a_{R}\right]+b \odot\left[q_{L}, q_{R}\right]\right) & t_{2} \leq t \leq t_{3} \\ -p^{-\epsilon}\left(\left[a_{L}, a_{R}\right]+b \odot\left[q_{L}, q_{R}\right]\right) & t_{3} \leq t \leq T\end{cases}
$$

Thus the above system is equivalent to

$$
\begin{aligned}
& \frac{d q_{L}}{d t}= \begin{cases}-a_{L} p^{-\epsilon} & 0 \leq t \leq t_{1} \\
(\mu-1) a_{R} p^{-\epsilon} & t_{1} \leq t \leq t_{2} \\
(\mu-1) p^{-\epsilon}\left(a_{R}+b q_{R}\right) & t_{2} \leq t \leq t_{3} \\
-p^{-\epsilon}\left(a_{L}+b q_{L}\right) & t_{3} \leq t \leq T\end{cases} \\
& \frac{d q_{R}}{d t}= \begin{cases}-a_{R} p^{-\epsilon} & 0 \leq t \leq t_{1} \\
(\mu-1) a_{L} p^{-\epsilon} & t_{1} \leq t \leq t_{2} \\
(\mu-1) p^{-\epsilon}\left(a_{L}+b q_{L}\right) & t_{2} \leq t \leq t_{3} \\
-p^{-\epsilon}\left(a_{R}+b q_{R}\right) & t_{3} \leq t \leq T\end{cases}
\end{aligned}
$$

After solving using the boundary conditions, $q(0)=q\left(t_{2}\right)=q(T)=0$ and $q\left(t_{2}\right)=Q_{m}$, we get

$$
\begin{gathered}
q_{L}(t)= \begin{cases}-a_{L} p^{-\epsilon} t & 0 \leq t \leq t_{1} \\
(\mu-1) a_{R} p^{-\epsilon}\left(t-t_{2}\right) & t_{1} \leq t \leq t_{2} \\
c_{1} e^{b k t}+c_{2} e^{-b k t}-\frac{a_{L}}{b} & t_{2} \leq t \leq t_{3} \\
k_{1} e^{r_{2} t}+k_{2} e^{-r_{2} t}+\frac{a_{L}}{b} & t_{3} \leq t \leq T\end{cases} \\
q_{R}(t)= \begin{cases}-a_{R} p^{-\epsilon} t & 0 \leq t \leq t_{1} \\
(\mu-1) a_{L} p^{-\epsilon}\left(t-t_{2}\right) & t_{1} \leq t \leq t_{2} \\
c_{1} e^{b k t}-c_{2} e^{-b k t}-\frac{a_{R}}{b} & t_{2} \leq t \leq t_{3} \\
-k_{1} e^{r_{2} t}+k_{2} e^{-r_{2} t}+\frac{a_{R}}{b} & t_{3} \leq t \leq T\end{cases}
\end{gathered}
$$

Where $k=(\mu-1) p^{-\epsilon}, r_{2}=-b p^{-\epsilon}$

$$
\begin{aligned}
& c_{1}=\frac{1}{e^{2 b k t_{3}}-e^{2 b k t_{2}}}\left[\left(Q_{m}+\frac{a_{L}}{b}\right) e^{b k t_{3}}-\frac{a_{L}}{b} e^{b k t_{2}}\right] \\
& c_{2}=\frac{a_{L}}{b} e^{b k t_{2}}-\frac{1}{e^{2 b k\left(t_{3}-t_{2}\right)}}\left[\left(Q_{m}+\frac{a_{L}}{b}\right) e^{b k t_{3}}-\frac{a_{L}}{b} e^{b k t_{2}}\right] \\
& k_{1}=\frac{a_{R}-a_{L}}{2 b} e^{-c_{2} T} \\
& k_{2}=-\frac{a_{L}+a_{R}}{2 b} e^{c_{2} T} \\
& a_{L}(\alpha)=\frac{\left(1+\theta_{r}\right) r_{1}+\left(r_{2}-r_{1}-2 \theta_{r} r_{1}\right) \alpha}{\left(1+\theta_{r}\right)-2 \theta_{r} \alpha} \\
& a_{R}(\alpha)=\frac{\left(1+\theta_{r}\right) r_{4}-\left(r_{4}-r_{3}+2 \theta_{r} r_{4}\right) \alpha}{\left(1+\theta_{r}\right)-2 \theta_{r} \alpha}
\end{aligned}
$$

Various costs related to the above system are

Total obsolescence cost of inventory 


$$
\begin{aligned}
& \text { TOC } C_{L}=\alpha^{\prime}\left(C_{s}-C_{s}^{\prime}\right)\left[\int_{t_{2}}^{t_{3}} q_{L}(t) d t+\int_{t_{3}}^{T} q_{L}(t) d t\right] \\
& =\alpha^{\prime}\left(C_{s}-C_{s}^{\prime}\right)\left[\int_{t_{2}}^{t_{3}}\left[c_{1} e^{b k t}+c_{2} e^{-b k t}-\frac{a_{L}}{b}\right] d t+\int_{t_{3}}^{T}\left[k_{1} e^{r_{2} t}+k_{2} e^{-r_{2} t}+\frac{a_{L}}{b}\right] d t\right] \\
& =\alpha^{\prime}\left(C_{s}-C_{s}^{\prime}\right)\left[\frac{c_{1}}{b k}\left(e^{b k t_{3}}-e^{b k t_{2}}\right)-\frac{c_{2}}{b k}\left(e^{-b k t_{3}}-e^{-b k t_{2}}\right)-\frac{a_{L}}{b}\left(t_{3}-t_{2}\right)+\frac{k_{1}}{r_{2}}\left(e^{r_{2} T}-e^{r_{2} t_{3}}\right)-\right. \\
& \left.\frac{k_{2}}{r_{2}}\left(e^{-r_{2} T}-e^{-r_{2} t_{3}}\right)+\frac{a_{L}}{b}\left(T-t_{3}\right)\right] \\
& T O C_{R}=\alpha^{\prime}\left(C_{s}-C_{s}^{\prime}\right)\left[\int_{t_{2}}^{t_{3}} q_{R}(t) d t+\int_{t_{3}}^{T} q_{R}(t) d t\right] \\
& =\alpha^{\prime}\left(C_{s}-C_{s}^{\prime}\right)\left[\int_{t_{2}}^{t_{3}}\left[c_{1} e^{b k t}-c_{2} e^{-b k t}-\frac{a_{R}}{b}\right] d t+\int_{t_{3}}^{T}\left[-k_{1} e^{r_{2} t}+k_{2} e^{-r_{2} t}+\frac{a_{R}}{b}\right] d t\right] \\
& =\alpha^{\prime}\left(C_{s}-C_{s}^{\prime}\right)\left[\frac{c_{1}}{b k}\left(e^{b k t_{3}}-e^{b k t_{2}}\right)+\frac{c_{2}}{b k}\left(e^{-b k t_{3}}-e^{-b k t_{2}}\right)-\frac{a_{R}}{b}\left(t_{3}-t_{2}\right)-\frac{k_{1}}{r_{2}}\left(e^{r_{2} T}-e^{r_{2} t_{3}}\right)-\right. \\
& \left.\frac{k_{2}}{r_{2}}\left(e^{-r_{2} T}-e^{-r_{2} t_{3}}\right)+\frac{a_{R}}{b}\left(T-t_{3}\right)\right]
\end{aligned}
$$

Total cost of emission of inventory obsolescence

$$
\begin{aligned}
& T E O_{L}=\alpha^{\prime} a^{\prime} C_{o c}\left[\int_{t_{2}}^{t_{3}} q_{L}(t) d t+\int_{t_{3}}^{T} q_{L}(t) d t\right] \\
& =\alpha^{\prime} a^{\prime} C_{o c}\left[\int_{t_{2}}^{t_{3}}\left[c_{1} e^{b k t}+c_{2} e^{-b k t}-\frac{a_{L}}{b}\right] d t+\int_{t_{3}}^{T}\left[k_{1} e^{r_{2} t}+k_{2} e^{-r_{2} t}+\frac{a_{L}}{b}\right] d t\right] \\
& =\alpha^{\prime} a^{\prime} C_{o c}\left[\frac{c_{1}}{b k}\left(e^{b k t_{3}}-e^{b k t_{2}}\right)-\frac{c_{2}}{b k}\left(e^{-b k t_{3}}-e^{-b k t_{2}}\right)-\frac{a_{L}}{b}\left(t_{3}-t_{2}\right)+\frac{k_{1}}{r_{2}}\left(e^{r_{2} T}-e^{r_{2} t_{3}}\right)-\frac{k_{2}}{r_{2}}\left(e^{-r_{2} T}-\right.\right. \\
& \left.\left.e^{-r_{2} t_{3}}\right)+\frac{a_{L}}{b}\left(T-t_{3}\right)\right] \\
& T E O_{R}=\alpha^{\prime} a^{\prime} C_{o c}\left[\int_{t_{2}}^{t_{3}} q_{R}(t) d t+\int_{t_{3}}^{T} q_{R}(t) d t\right] \\
& =\alpha^{\prime} a^{\prime} C_{o c}\left[\int_{t_{2}}^{t_{3}}\left[c_{1} e^{b k t}-c_{2} e^{-b k t}-\frac{a_{R}}{b}\right] d t+\int_{t_{3}}^{T}\left[-k_{1} e^{r_{2} t}+k_{2} e^{-r_{2} t}+\frac{a_{R}}{b}\right] d t\right] \\
& =\alpha^{\prime} a^{\prime} C_{o c}\left[\frac{c_{1}}{b k}\left(e^{b k t_{3}}-e^{b k t_{2}}\right)+\frac{c_{2}}{b k}\left(e^{-b k t_{3}}-e^{-b k t_{2}}\right)-\frac{a_{R}}{b}\left(t_{3}-t_{2}\right)-\frac{k_{1}}{r_{2}}\left(e^{r_{2} T}-e^{r_{2} t_{3}}\right)-\frac{k_{2}}{r_{2}}\left(e^{-r_{2} T}-\right.\right. \\
& \left.\left.e^{-r_{2} t_{3}}\right)+\frac{a_{R}}{b}\left(T-t_{3}\right)\right]
\end{aligned}
$$

Total backordering cost

$T B C_{L}=C_{b}\left[\int_{0}^{t_{1}} q_{L}(t) d t+\int_{t_{1}}^{t_{2}} q_{L}(t) d t\right]=c_{b}\left[-\frac{1}{2} a_{L} p^{-\epsilon} t_{1}^{2}-\frac{1}{2}(\mu-1) a_{R} p^{-\epsilon}\left(t_{2}-t_{1}\right)^{2}\right]$

$T B C_{R}=C_{b}\left[\int_{0}^{t_{1}} q_{R}(t) d t+\int_{t_{1}}^{t_{2}} q_{R}(t) d t\right]=c_{b}\left[-\frac{1}{2} a_{R} p^{-\epsilon} t_{1}^{2}-\frac{1}{2}(\mu-1) a_{L} p^{-\epsilon}\left(t_{2}-t_{1}\right)^{2}\right]$

Total goodwill loss for back-order is

$T G C_{L}=C_{g}\left[\int_{0}^{t_{1}} q_{L}(t) d t+\int_{t_{1}}^{t_{2}} q_{L}(t) d t\right]=c_{g}\left[-\frac{1}{2} a_{L} p^{-\epsilon} t_{1}^{2}-\frac{1}{2}(\mu-1) a_{R} p^{-\epsilon}\left(t_{2}-t_{1}\right)^{2}\right]$

$T G C_{R}=C_{g}\left[\int_{0}^{t_{1}} q_{R}(t) d t+\int_{t_{1}}^{t_{2}} q_{R}(t) d t\right]=c_{g}\left[-\frac{1}{2} a_{R} p^{-\epsilon} t_{1}^{2}-\frac{1}{2}(\mu-1) a_{L} p^{-\epsilon}\left(t_{2}-t_{1}\right)^{2}\right]$

Total holding cost

TH $C_{L}=\int_{t_{2}}^{t_{3}} C_{h} q_{L}(t) d t+\int_{t_{3}}^{T} C_{h} q_{L}(t) d t$

$=C_{h}\left[\frac{c_{1}}{b k}\left(e^{b k t_{3}}-e^{b k t_{2}}\right)-\frac{c_{2}}{b k}\left(e^{-b k t_{3}}-e^{-b k t_{2}}\right)-\frac{a_{L}}{b}\left(t_{3}-t_{2}\right)+\frac{k_{1}}{r_{2}}\left(e^{r_{2} T}-e^{r_{2} t_{3}}\right)-\frac{k_{2}}{r_{2}}\left(e^{-r_{2} T}-\right.\right.$

$\left.\left.e^{-r_{2} t_{3}}\right)+\frac{a_{L}}{b}\left(T-t_{3}\right)\right]$

$T H C_{R}=\int_{t_{2}}^{t_{3}} C_{h} q_{R}(t) d t+\int_{t_{3}}^{T} C_{h} q_{R}(t) d t$

$=C_{h}\left[\frac{c_{1}}{b k}\left(e^{b k t_{3}}-e^{b k t_{2}}\right)+\frac{c_{2}}{b k}\left(e^{-b k t_{3}}-e^{-b k t_{2}}\right)-\frac{a_{R}}{b}\left(t_{3}-t_{2}\right)-\frac{k_{1}}{r_{2}}\left(e^{r_{2} T}-e^{r_{2} t_{3}}\right)-\frac{k_{2}}{r_{2}}\left(e^{-r_{2} T}-\right.\right.$ $\left.\left.e^{-r_{2} t_{3}}\right)+\frac{a_{R}}{b}\left(T-t_{3}\right)\right]$

Total emission cost of carbon for holding inventory

$$
\begin{aligned}
& T E H_{L}=C_{h c} b^{\prime}\left[\int_{t_{2}}^{t_{3}} q_{L}(t) d t+\int_{t_{3}}^{T} q_{L}(t) d t\right] \\
& =C_{h c} b^{\prime}\left[\frac{c_{1}}{b k}\left(e^{b k t_{3}}-e^{b k t_{2}}\right)-\frac{c_{2}}{b k}\left(e^{-b k t_{3}}-e^{-b k t_{2}}\right)-\frac{a_{L}}{b}\left(t_{3}-t_{2}\right)+\frac{k_{1}}{r_{2}}\left(e^{r_{2} T}-e^{r_{2} t_{3}}\right)-\frac{k_{2}}{r_{2}}\left(e^{-r_{2} T}-\right.\right. \\
& \left.\left.e^{-r_{2} t_{3}}\right)+\frac{a_{L}}{b}\left(T-t_{3}\right)\right]
\end{aligned}
$$




$$
\begin{aligned}
& T E H_{R}=C_{h c} b^{\prime}\left[\int_{t_{2}}^{t_{3}} q_{R}(t) d t+\int_{t_{3}}^{T} q_{R}(t) d t\right] \\
& =C_{h c} b^{\prime}\left[\frac{c_{1}}{b k}\left(e^{b k t_{3}}-e^{b k t_{2}}\right)+\frac{c_{2}}{b k}\left(e^{-b k t_{3}}-e^{-b k t_{2}}\right)-\frac{a_{R}}{b}\left(t_{3}-t_{2}\right)-\frac{k_{1}}{r_{2}}\left(e^{r_{2} T}-e^{r_{2} t_{3}}\right)-\frac{k_{2}}{r_{2}}\left(e^{-r_{2} T}-\right.\right. \\
& \left.\left.e^{-r_{2} t_{3}}\right)+\frac{a_{R}}{b}\left(T-t_{3}\right)\right]
\end{aligned}
$$

Total set-up cost $=C_{3}$

Total revenue earned

$T R E_{L}=c_{S}\left[\mu a_{L} p^{-\epsilon}\left(t_{2}-t_{1}\right)+\mu \int_{t_{2}}^{t_{3}} p^{-\epsilon}\left(a_{L}+b q_{L}\right) d t\right]$

$=c_{S}\left[\mu a_{L} p^{-\epsilon}\left(t_{2}-t_{1}\right)+\frac{\mu p^{-\epsilon}}{k}\left\{c_{1}\left(e^{b k t_{3}}-e^{b k t_{2}}\right)-c_{2}\left(e^{-b k t_{3}}-e^{-b k t_{2}}\right)\right\}\right]$

$T R E_{R}=c_{S}\left[\mu a_{R} p^{-\epsilon}\left(t_{2}-t_{1}\right)+\mu \int_{t_{2}}^{t_{3}} p^{-\epsilon}\left(a_{R}+b q_{R}\right) d t\right]$

$=c_{S}\left[\mu a_{R} p^{-\epsilon}\left(t_{2}-t_{1}\right)+\frac{\mu p^{-\epsilon}}{k}\left\{c_{1}\left(e^{b k t_{3}}-e^{b k t_{2}}\right)+c_{2}\left(e^{-b k t_{3}}-e^{-b k t_{2}}\right)\right\}\right]$

Total Production cost

$T P C_{L}=p\left[\mu a_{L} p^{-\epsilon}\left(t_{2}-t_{1}\right)+\mu \int_{t_{2}}^{t_{3}} p^{-\epsilon}\left(a_{L}+b q_{L}\right) d t\right]$

$=p\left[\mu a_{L} p^{-\epsilon}\left(t_{2}-t_{1}\right)+\frac{\mu p^{-\epsilon}}{k}\left\{c_{1}\left(e^{b k t_{3}}-e^{b k t_{2}}\right)-c_{2}\left(e^{-b k t_{3}}-e^{-b k t_{2}}\right)\right\}\right]$

$T P C_{R}=p\left[\mu a_{R} p^{-\epsilon}\left(t_{2}-t_{1}\right)+\mu \int_{t_{2}}^{t_{3}} p^{-\epsilon}\left(a_{R}+b q_{R}\right) d t\right]$

$=p\left[\mu a_{R} p^{-\epsilon}\left(t_{2}-t_{1}\right)+\frac{\mu p^{-\epsilon}}{k}\left\{c_{1}\left(e^{b k t_{3}}-e^{b k t_{2}}\right)+c_{2}\left(e^{-b k t_{3}}-e^{-b k t_{2}}\right)\right\}\right]$

The emission cost of carbon for manufacturing total units

$T E P_{L}=c_{m c}\left[\mu a_{L} p^{-\epsilon}\left(t_{2}-t_{1}\right)+\mu \int_{t_{2}}^{t_{3}} p^{-\epsilon}\left(a_{L}+b q_{L}\right) d t\right]$

$=c_{m c}\left[\mu a_{L} p^{-\epsilon}\left(t_{2}-t_{1}\right)+\frac{\mu p^{-\epsilon}}{k}\left\{c_{1}\left(e^{b k t_{3}}-e^{b k t_{2}}\right)-c_{2}\left(e^{-b k t_{3}}-e^{-b k t_{2}}\right)\right\}\right]$

$T E P_{R}=c_{m c}\left[\mu a_{R} p^{-\epsilon}\left(t_{2}-t_{1}\right)+\mu \int_{t_{2}}^{t_{3}} p^{-\epsilon}\left(a_{R}+b q_{R}\right) d t\right]$

$=c_{m c}\left[\mu a_{R} p^{-\epsilon}\left(t_{2}-t_{1}\right)+\frac{\mu p^{-\epsilon}}{k}\left\{c_{1}\left(e^{b k t_{3}}-e^{b k t_{2}}\right)+c_{2}\left(e^{-b k t_{3}}-e^{-b k t_{2}}\right)\right\}\right]$

Therefore three sub-cases may arise depending upon the values of changing time periods.

Sub-case 2.1: $0 \leq t_{1} \leq N \leq M \leq t_{2} \leq t_{3} \leq T$

In this case if the amount is paid within $M$ by the retailer, then there is no interest payable. Otherwise, the retailer will pay for the rest of the inventory. In this case the total amount of interest paid and interest earned by the retailer is calculated.

Hence, total interest paid

$$
\begin{aligned}
& \text { TI } P_{L}=p i_{p}\left[\int_{M}^{t_{2}}\left(1+T-t_{2}\right) q_{L}(t) d t+\int_{t_{2}}^{t_{3}}\left(1+T-t_{3}\right) q_{L}(t) d t+\int_{t_{3}}^{T} q_{L}(t) d t\right] \\
& =p i_{p}\left[\frac{1}{2}\left(1+T-t_{2}\right)(\mu-1) a_{L} p^{-\epsilon}\left(t_{2}-M\right)^{2}+\left(1+T-t_{3}\right)\left\{\frac{c_{1}}{b k}\left(e^{b k t_{3}}-e^{b k t_{2}}\right)-\frac{c_{2}}{b k}\left(e^{-b k t_{3}}-\right.\right.\right. \\
& \left.\left.\left.e^{-b k t_{2}}\right)-\frac{a_{L}}{b}\left(t_{3}-t_{2}\right)\right\}+\frac{k_{1}}{r_{2}}\left(e^{r_{2} T}-e^{r_{2} t_{3}}\right)-\frac{k_{2}}{r_{2}}\left(e^{-r_{2} T}-e^{-r_{2} t_{3}}\right)+\frac{a_{L}}{b}\left(T-t_{3}\right)\right] \\
& \quad T I P_{R}=p i_{p}\left[\int_{M}^{t_{2}}\left(1+T-t_{2}\right) q_{R}(t) d t+\int_{t_{2}}^{t_{3}}\left(1+T-t_{3}\right) q_{R}(t) d t+\int_{t_{3}}^{T} q_{R}(t) d t\right] \\
& T I P_{R}=p i_{p}\left[\frac{1}{2}\left(1+T-t_{2}\right)(\mu-1) a_{R} p^{-\epsilon}\left(t_{2}-M\right)^{2}+\left(1+T-t_{3}\right)\left\{\frac{c_{1}}{b k}\left(e^{b k t_{3}}-e^{b k t_{2}}\right)+\frac{c_{2}}{b k}\left(e^{-b k t_{3}}-\right.\right.\right. \\
& \left.\left.\left.e^{-b k t_{2}}\right)-\frac{a_{R}}{b}\left(t_{3}-t_{2}\right)\right\}-\frac{k_{1}}{r_{2}}\left(e^{r_{2} T}-e^{r_{2} t_{3}}\right)-\frac{k_{2}}{r_{2}}\left(e^{-r_{2} T}-e^{-r_{2} t_{3}}\right)+\frac{a_{R}}{b}\left(T-t_{3}\right)\right]
\end{aligned}
$$

And, total interest earned

$T I E_{L}=C_{s} i_{e}\left[(T-N) \int_{t_{1}}^{N} D_{L}(p, q) d t+(1+T-M) \int_{N}^{M} D_{L}(p, q)(M-t) d t+(1+T-\right.$ $\left.\left.t_{2}\right) \int_{M}^{t_{2}} D_{L}(p, q)\left(t_{2}-t\right) d t+\left(1+T-t_{3}\right) \int_{t_{2}}^{t_{3}} D_{L}(p, q)\left(t_{3}-t\right) d t+\int_{t_{3}}^{T} D_{L}(p, q)(T-t) d t\right]$

$$
\begin{aligned}
& =C_{s} i_{e}\left[(T-N) a_{L} p^{-\epsilon}\left(N-t_{1}\right)+\frac{1}{2}(1+T-M) a_{L} p^{-\epsilon}(M-N)^{2}+\frac{1}{2}\left(1+T-t_{2}\right) a_{L} p^{-\epsilon}\left(t_{2}-\right.\right. \\
& M)^{2}+p^{-\epsilon} t_{3} b\left(1+T-t_{3}\right)\left\{\frac{c_{1}}{b k}\left(e^{b k t_{3}}-e^{b k t_{2}}\right)-\frac{c_{2}}{b k}\left(e^{-b k t_{3}}-e^{-b k t_{2}}\right)\right\}-p^{-\epsilon} b\left(1+T-t_{3}\right)\left\{\frac { 1 } { b k } \left(t_{3} e^{b k t_{3}}-\right.\right. \\
& \left.\left.t_{2} e^{b k t_{2}}\right)-\frac{1}{b^{2} k^{2}}\left(e^{b k t_{3}}-e^{b k t_{2}}\right)\right\}+T\left\{\frac{b k_{1}}{r_{2}}\left(e^{r_{2} T}-e^{r_{2} t_{3}}\right)-\frac{b k_{2}}{r_{2}}\left(e^{-r_{2} T}-e^{-r_{2} t_{3}}\right)+2 a_{L}\left(T-t_{3}\right)\right\}-
\end{aligned}
$$




$$
\begin{aligned}
& b k_{1}\left\{\frac{1}{r_{2}}\left(T e^{r_{2} T}-t_{3} e^{r_{2} t_{3}}\right)-\frac{1}{r_{2}^{2}}\left(e^{r_{2} T}-e^{r_{2} t_{3}}\right)\right\}-b k_{2}\left\{-\frac{1}{r_{2}}\left(T e^{-r_{2} T}-t_{3} e^{-r_{2} t_{3}}\right)-\frac{1}{r_{2}^{2}}\left(e^{-r_{2} T}-\right.\right. \\
& \left.\left.\left.e^{-r_{2} t_{3}}\right)\right\}-2 a_{L}\left(T-t_{3}\right)\right] \\
& T I E_{R}=C_{s} i_{e}\left[(T-N) \int_{t_{1}}^{N} D_{R}(p, q) d t+(1+T-M) \int_{N}^{M} D_{R}(p, q)(M-t) d t+(1+T-\right. \\
& \left.\left.t_{2}\right) \int_{M}^{t_{2}} D_{R}(p, q)\left(t_{2}-t\right) d t+\left(1+T-t_{3}\right) \int_{t_{2}}^{t_{3}} D_{R}(p, q)\left(t_{3}-t\right) d t+\int_{t_{3}}^{T} D_{R}(p, q)(T-t) d t\right] \\
& \quad=C_{s} i_{e}\left[(T-N) a_{R} p^{-\epsilon}\left(N-t_{1}\right)+\frac{1}{2}(1+T-M) a_{R} p^{-\epsilon}(M-N)^{2}+\frac{1}{2}\left(1+T-t_{2}\right) a_{R} p^{-\epsilon}\left(t_{2}-\right.\right. \\
& M)^{2}+p^{-\epsilon} t_{3} b\left(1+T-t_{3}\right)\left\{\frac{c_{1}}{b k}\left(e^{b k t_{3}}-e^{b k t_{2}}\right)+\frac{c_{2}}{b k}\left(e^{-b k t_{3}}-e^{-b k t_{2}}\right)\right\}-p^{-\epsilon} b\left(1+T-t_{3}\right)\left\{\frac { 1 } { b k } \left(t_{3} e^{b k t_{3}}-\right.\right. \\
& \left.\left.\left.t_{2} e^{b k t_{2}}\right)-\frac{1}{b^{2} k^{2}}\left(e^{b k t_{3}}-e^{b k t_{2}}\right)\right\}+T\left\{\frac{b k_{1}}{r_{2}}\left(e^{r_{2} T}-e^{r_{2} t_{3}}\right)-\frac{b k_{2}}{r_{2}}\left(e^{-r_{2} T}-e^{-r_{2} t_{3}}\right)+2 a_{R}\left(T-t_{3}\right)\right\}\right\}+ \\
& b k_{1}\left\{\frac{1}{r_{2}}\left(T e^{r_{2} T}-t_{3} e^{r_{2} t_{3}}\right)-\frac{1}{r_{2}^{2}}\left(e^{r_{2} T}-e^{r_{2} t_{3}}\right)\right\}-b k_{2}\left\{-\frac{1}{r_{2}}\left(T e^{-r_{2} T}-t_{3} e^{-r_{2} t_{3}}\right)-\frac{1}{r_{2}^{2}}\left(e^{-r_{2} T}-\right.\right. \\
& \left.\left.\left.e^{-r_{2} t_{3}}\right)\right\}-2 a_{L}\left(T-t_{3}\right)\right]
\end{aligned}
$$

Sub-case 2.2: $0 \leq t_{1} \leq t_{2} \leq N \leq M \leq t_{3} \leq T$

In this case the total amount of interest paid and interest earned by the retailer is calculated.

Hence, total interest paid

$$
\begin{aligned}
& T I P_{L}=p i_{p}\left[\int_{M}^{t_{3}}\left(1+T-t_{3}\right) q_{L}(t) d t+\int_{t_{3}}^{T} q_{L}(t) d t\right] \\
& =p i_{p}\left[\left(1+T-t_{3}\right)\left\{\frac{c_{1}}{b k}\left(e^{b k t_{3}}-e^{b k M}\right)-\frac{c_{2}}{b k}\left(e^{-b k t_{3}}-e^{-b k M}\right)-\frac{a_{L}}{b}\left(t_{3}-M\right)\right\}+\frac{k_{1}}{r_{2}}\left(e^{r_{2} T}-e^{r_{2} t_{3}}\right)-\right. \\
& \left.\frac{k_{2}}{r_{2}}\left(e^{-r_{2} T}-e^{-r_{2} t_{3}}\right)+\frac{a_{L}}{b}\left(T-t_{3}\right)\right] \\
& \quad T I P_{R}=p i_{p}\left[\int_{M}^{t_{3}}\left(1+T-t_{3}\right) q_{R}(t) d t+\int_{t_{3}}^{T} q_{R}(t) d t\right] \\
& =p i_{p}\left[\left(1+T-t_{3}\right)\left\{\frac{c_{1}}{b k}\left(e^{b k t_{3}}-e^{b k M}\right)+\frac{c_{2}}{b k}\left(e^{-b k t_{3}}-e^{-b k M}\right)-\frac{a_{R}}{b}\left(t_{3}-M\right)\right\}-\frac{k_{1}}{r_{2}}\left(e^{r_{2} T}-e^{r_{2} t_{3}}\right)-\right. \\
& \left.\frac{k_{2}}{r_{2}}\left(e^{-r_{2} T}-e^{-r_{2} t_{3}}\right)+\frac{a_{R}}{b}\left(T-t_{3}\right)\right]
\end{aligned}
$$

Total interest earned

$$
\begin{aligned}
& T I E_{L}=C_{s} i_{e}\left[\left(T-t_{2}\right) \int_{t_{1}}^{t_{2}} D_{L}(p, q) d t+\left(1+T-t_{3}\right) \int_{t_{2}}^{t_{3}} D_{L}(p, q)\left(t_{3}-t\right) d t+\int_{t_{3}}^{T} D_{L}(p, q)(T-t) d t\right] \\
& =C_{s} i_{e}\left[a_{L} p^{-\epsilon}\left(T-t_{2}\right)\left(t_{2}-t_{1}\right)+\left(1+T-t_{3}\right) p^{-\epsilon} b t_{3}\left\{\frac{c_{1}}{k}\left(e^{b k t_{3}}-e^{b k t_{2}}\right)-\frac{c_{2}}{k}\left(e^{-b k t_{3}}-\right.\right.\right. \\
& \left.\left.e^{-b k t_{2}}\right)\right\}-p^{-\epsilon}\left(1+T-t_{3}\right)\left[c_{1} b\left\{\frac{1}{b k}\left(t_{3} e^{b k t_{3}}-t_{2} e^{b k t_{2}}\right)-\frac{1}{b^{2} k^{2}}\left(e^{b k t_{3}}-e^{b k t_{2}}\right)\right\}-c_{2} b\left\{-\frac{1}{b k}\left(t_{3} e^{-b k t_{3}}-\right.\right.\right. \\
& \left.\left.\left.t_{2} e^{-b k t_{2}}\right)-\frac{1}{b^{2} k^{2}}\left(e^{-b k t_{3}}-e^{-b k t_{2}}\right)\right\}\right]+T\left\{-\frac{k_{1}}{r_{2}}\left(e^{r_{2} T}-e^{r_{2} t_{3}}\right)-\frac{k_{2}}{r_{2}}\left(e^{-r_{2} T}-e^{-r_{2} t_{3}}\right)+\frac{2 a_{R}}{b}(T-\right. \\
& \left.\left.t_{3}\right)\right\}-k_{1}\left\{-\frac{1}{r_{2}}\left(T e^{r_{2} T}-t_{3} e^{r_{2} t_{3}}\right)+\frac{1}{r_{2}^{2}}\left(e^{r_{2} T}-e^{r_{2} t_{3}}\right)\right\}-k_{2}\left\{-\frac{1}{r_{2}}\left(T e^{-r_{2} T}-t_{3} e^{-r_{2} t_{3}}\right)-\frac{1}{r_{2}^{2}}\left(e^{-r_{2} T}-\right.\right. \\
& \left.\left.\left.e^{-r_{2} t_{3}}\right)-\frac{a_{R}}{b}\left(T^{2}-t_{3}^{2}\right)\right\}\right] \\
& T I E_{R}=C_{s} i_{e}\left[\left(T-t_{2}\right) \int_{t_{1}}^{t_{2}} D_{R}(p, q) d t+\left(1+T-t_{3}\right) \int_{t_{2}}^{t_{3}} D_{R}(p, q)\left(t_{3}-t\right) d t+\int_{t_{3}}^{T} D_{R}(p, q)(T-\right. \\
& =C_{s} i_{e}\left[a_{R} p^{-\epsilon}\left(T-t_{2}\right)\left(t_{2}-t_{1}\right)+\left(1+T-t_{3}\right) p^{-\epsilon} b t_{3}\left\{\frac{c_{1}}{k}\left(e^{b k t_{3}}-e^{b k t_{2}}\right)+\frac{c_{2}}{k}\left(e^{-b k t_{3}}-\right.\right.\right. \\
& \left.\left.e^{-b k t_{2}}\right)\right\}-p^{-\epsilon}\left(1+T-t_{3}\right)\left[c_{1} b\left\{\frac{1}{b k}\left(t_{3} e^{b k t_{3}}-t_{2} e^{b k t_{2}}\right)-\frac{1}{b^{2} k^{2}}\left(e^{b k t_{3}}-e^{b k t_{2}}\right)\right\}+c_{2} b\left\{-\frac{1}{b k}\left(t_{3} e^{-b k t_{3}}-\right.\right.\right. \\
& \left.\left.\left.t_{2} e^{-b k t_{2}}\right)-\frac{1}{b^{2} k^{2}}\left(e^{-b k t_{3}}-e^{-b k t_{2}}\right)\right\}\right]+T\left\{\frac{k_{1}}{r_{2}}\left(e^{r_{2} T}-e^{r_{2} t_{3}}\right)-\frac{k_{2}}{r_{2}}\left(e^{-r_{2} T}-e^{-r_{2} t_{3}}\right)+\frac{2 a_{R}}{b}(T-\right. \\
& \left.\left.t_{3}\right)\right\}+k_{1}\left\{-\frac{1}{r_{2}}\left(T e^{r_{2} T}-t_{3} e^{r_{2} t_{3}}\right)+\frac{1}{r_{2}^{2}}\left(e^{r_{2} T}-e^{r_{2} t_{3}}\right)\right\}-k_{2}\left\{-\frac{1}{r_{2}}\left(T e^{-r_{2} T}-t_{3} e^{-r_{2} t_{3}}\right)-\frac{1}{r_{2}^{2}}\left(e^{-r_{2} T}-\right.\right. \\
& \left.\left.\left.e^{-r_{2} t_{3}}\right)-\frac{a_{R}}{b}\left(T^{2}-t_{3}^{2}\right)\right\}\right]
\end{aligned}
$$

Sub-case 2.3: $0 \leq t_{1} \leq t_{2} \leq N \leq t_{3} \leq T \leq M$

In this case interest payable by the retailer is zero, i.e., $T I P_{L}=T I P_{R}=0$

But, interest earned by the retailer is given by 


$$
\begin{aligned}
& T I E_{L}=C_{s} i_{e}\left[\left(M-t_{2}\right) \int_{t_{1}}^{t_{2}} D_{L}(p, q) d t+(M-N) \int_{t_{2}}^{N} D_{L}(p, q) d t\right. \\
& \left.+\left(1+M-t_{3}\right) \int_{N}^{t_{3}} D_{L}(p, q)\left(t_{3}-t\right) d t+(1+M-T) \int_{t_{3}}^{T} D_{L}(p, q)(T-t) d t\right] \\
& =C_{s} i_{e} p^{-\epsilon}\left[a_{L}\left(M-t_{2}\right)\left(t_{2}-t_{1}\right)+b(M-N)\left\{\frac{c_{1}}{b k}\left(e^{b k N}-e^{b k t_{2}}\right)-\frac{c_{2}}{b k}\left(e^{-b k N}-e^{-b k t_{2}}\right)\right\}+(1+\right. \\
& \left.M-t_{3}\right) t_{3}\left\{\frac{c_{1}}{b k}\left(e^{b k t_{3}}-e^{b k N}\right)-\frac{c_{2}}{b k}\left(e^{-b k t_{3}}-e^{-b k N}\right)\right\}+\left(1+M-t_{3}\right)\left[c _ { 1 } \left\{\frac{1}{b k}\left(t_{3} e^{b k t_{3}}-N e^{b k N}\right)-\right.\right. \\
& \left.\left.\frac{1}{b^{2} k^{2}}\left(e^{b k t_{3}}-e^{b k N}\right)\right\}+c_{2}\left\{-\frac{1}{b k}\left(t_{3} e^{-b k t_{3}}-N e^{-b k N}\right)-\frac{1}{b^{2} k^{2}}\left(e^{-b k t_{3}}-e^{-b k N}\right)\right\}\right]+(1+M- \\
& T) T\left\{\frac{b k_{1}}{r_{2}}\left(e^{r_{2} T}-e^{r_{2} t_{3}}\right)-\frac{b k_{2}}{r_{2}}\left(e^{-r_{2} T}-e^{-r_{2} t_{3}}\right)+2 a_{L}\left(T-t_{3}\right)\right\}-(1+M-T)\left[b k _ { 1 } \left\{\frac { 1 } { r _ { 2 } } \left(T e^{r_{2} T}-\right.\right.\right. \\
& \left.\left.\left.\left.t_{3} e^{r_{2} t_{3}}\right)-\frac{1}{r_{2}^{2}}\left(e^{r_{2} T}-e^{r_{2} t_{3}}\right)\right\}+b k_{2}\left\{-\frac{1}{r_{2}}\left(T e^{-r_{2} T}-t_{3} e^{-r_{2} t_{3}}\right)-\frac{1}{r_{2}^{2}}\left(e^{-r_{2} T}-e^{-r_{2} t_{3}}\right)\right\}+a_{L}\left(T^{2}-t_{3}^{2}\right)\right]\right] \\
& T I E_{R}=C_{s} i_{e}\left[\left(M-t_{2}\right) \int_{t_{1}}^{t_{2}} D_{R}(p, q) d t+(M-N) \int_{t_{2}}^{N} D_{R}(p, q) d t\right. \\
& \left.+\left(1+M-t_{3}\right) \int_{N}^{t_{3}} D_{R}(p, q)\left(t_{3}-t\right) d t+(1+M-T) \int_{t_{3}}^{T} D_{R}(p, q)(T-t) d t\right] \\
& =C_{s} i_{e} p^{-\epsilon}\left[a_{R}\left(M-t_{2}\right)\left(t_{2}-t_{1}\right)+b(M-N)\left\{\frac{c_{1}}{b k}\left(e^{b k N}-e^{b k t_{2}}\right)+\frac{c_{2}}{b k}\left(e^{-b k N}-e^{-b k t_{2}}\right)\right\}+(1+\right. \\
& \left.M-t_{3}\right) t_{3}\left\{\frac{c_{1}}{b k}\left(e^{b k t_{3}}-e^{b k N}\right)+\frac{c_{2}}{b k}\left(e^{-b k t_{3}}-e^{-b k N}\right)\right\}+\left(1+M-t_{3}\right)\left[c _ { 1 } \left\{\frac{1}{b k}\left(t_{3} e^{b k t_{3}}-N e^{b k N}\right)-\right.\right. \\
& \left.\left.\frac{1}{b^{2} k^{2}}\left(e^{b k t_{3}}-e^{b k N}\right)\right\}-c_{2}\left\{-\frac{1}{b k}\left(t_{3} e^{-b k t_{3}}-N e^{-b k N}\right)-\frac{1}{b^{2} k^{2}}\left(e^{-b k t_{3}}-e^{-b k N}\right)\right\}\right]+(1+M- \\
& T) T\left\{-\frac{b k_{1}}{r_{2}}\left(e^{r_{2} T}-e^{r_{2} t_{3}}\right)-\frac{b k_{2}}{r_{2}}\left(e^{-r_{2} T}-e^{-r_{2} t_{3}}\right)+2 a_{R}\left(T-t_{3}\right)\right\}-(1+M-T)\left[-b k_{1}\left\{\frac { 1 } { r _ { 2 } } \left(T e^{r_{2} T}-\right.\right.\right. \\
& \left.\left.\left.\left.t_{3} e^{r_{2} t_{3}}\right)-\frac{1}{r_{2}^{2}}\left(e^{r_{2} T}-e^{r_{2} t_{3}}\right)\right\}+b k_{2}\left\{-\frac{1}{r_{2}}\left(T e^{-r_{2} T}-t_{3} e^{-r_{2} t_{3}}\right)-\frac{1}{r_{2}^{2}}\left(e^{-r_{2} T}-e^{-r_{2} t_{3}}\right)\right\}+a_{R}\left(T^{2}-t_{3}^{2}\right)\right]\right]
\end{aligned}
$$

In this case also the objective functions are defined as follows:

Max Profit

$$
(T P)=\frac{1}{T}\left[T R E+T I E-T P C-T H C-T O C-T I P-T B C-T G C-C_{3}\right]
$$

Min Emission $(T E)=T E O+T E H+T E P$

On taking $\alpha$-cut over the total profit and emission per unit time is a crisp interval and is defined by

$T P=\left[T P_{L}, T P_{R}\right]$ where,

$T P_{L}=\frac{1}{T}\left[T R E_{L}+T I E_{L}-T P C_{L}-T H C_{L}-T O C_{L}-T I P_{L}-T B C_{L}-T G C_{L}-C_{3}\right]$

$T P_{R}=\frac{1}{T}\left[T R E_{R}+T I E_{R}-T P C_{R}-T H C_{R}-T O C_{R}-T I P_{R}-T B C_{R}-T G C_{R}-C_{3}\right]$

And, $T E=\left[T E_{L}, T E_{R}\right]$ where,

$T E_{L}=T E O_{L}+T E H_{L}+T E P_{L}$

$T E_{R}=T E O_{R}+T E H_{R}+T E P_{R}$, when $0 \leq \alpha \leq 0.5$

Again, if $\alpha \in[0.5,1]$, then the system of interval fuzzy differential equation is given by

$$
\left[\frac{d q_{L}}{d t}, \frac{d q_{R}}{d t}\right]= \begin{cases}-\left[a_{L}, a_{R}\right] \odot p^{-\epsilon} & 0 \leq t \leq t_{1} \\ (\mu-1) \odot\left[a_{L}, a_{R}\right] \odot p^{-\epsilon} & t_{1} \leq t \leq t_{2} \\ (\mu-1) \odot p^{-\epsilon}\left(\left[a_{L}, a_{R}\right]+b \odot\left[q_{L}, q_{R}\right]\right) & t_{2} \leq t \leq t_{3} \\ -p^{-\epsilon}\left(\left[a_{L}, a_{R}\right]+b \odot\left[q_{L}, q_{R}\right]\right) & t_{3} \leq t \leq T\end{cases}
$$

Therefore two cases arise

Case 1: gH-(i) differentiability

Case 2: gH-(ii) differentiability

In case 1: gH-(i) differentiability, the above system reduces to

$$
\frac{d q_{L}}{d t}= \begin{cases}-a_{R} p^{-\epsilon} & 0 \leq t \leq t_{1} \\ (\mu-1) a_{L} p^{-\epsilon} & t_{1} \leq t \leq t_{2} \\ (\mu-1) p^{-\epsilon}\left(a_{L}+b q_{L}\right) & t_{2} \leq t \leq t_{3} \\ -p^{-\epsilon}\left(a_{R}+b q_{R}\right) & t_{3} \leq t \leq T\end{cases}
$$




$$
\frac{d q_{R}}{d t}= \begin{cases}-a_{L} p^{-\epsilon} & 0 \leq t \leq t_{1} \\ (\mu-1) a_{R} p^{-\epsilon} & t_{1} \leq t \leq t_{2} \\ (\mu-1) p^{-\epsilon}\left(a_{R}+b q_{R}\right) & t_{2} \leq t \leq t_{3} \\ -p^{-\epsilon}\left(a_{L}+b q_{L}\right) & t_{3} \leq t \leq T\end{cases}
$$

After solving using the boundary conditions, $q(0)=q\left(t_{2}\right)=q(T)=0$, we get

$$
\begin{gathered}
q_{L}(t)= \begin{cases}-a_{R} p^{-\epsilon} t & 0 \leq t \leq t_{1} \\
(\mu-1) a_{L} p^{-\epsilon}\left(t-t_{2}\right) & t_{1} \leq t \leq t_{2} \\
\frac{a_{L}}{b}\left[e^{x\left(t-t_{2}\right)}-1\right] & t_{2} \leq t \leq t_{3} \\
-\frac{a_{R}}{b}\left[e^{y(T-t)}-1\right] & t_{3} \leq t \leq T\end{cases} \\
q_{R}(t)= \begin{cases}-a_{L} p^{-\epsilon} t & 0 \leq t \leq t_{1} \\
(\mu-1) a_{R} p^{-\epsilon}\left(t-t_{2}\right) & t_{1} \leq t \leq t_{2} \\
\frac{a_{R}}{b}\left[e^{x\left(t-t_{2}\right)}-1\right] & t_{2} \leq t \leq t_{3} \\
-\frac{a_{L}}{b}\left[e^{y(T-t)}-1\right] & t_{3} \leq t \leq T\end{cases}
\end{gathered}
$$

where, $x=b(\mu-1) p^{-\epsilon}$ and $y=b p^{-\epsilon}$

$$
\begin{aligned}
& a_{L}(\alpha)=\frac{\left(r_{1}-\theta_{l} r_{2}\right)+\left(r_{2}-r_{1}-\theta_{l} r_{2}\right) \alpha}{\left(1-\theta_{l}\right)+2 \theta_{l} \alpha}, \\
& a_{R}(\alpha)=\frac{\left(r_{4}-\theta_{l} r_{3}\right)+\left(r_{4}-r_{3}-2 \theta_{l} r_{3}\right) \alpha}{\left(1-\theta_{l}\right)+2 \theta_{l} \alpha}
\end{aligned}
$$

In this case all the relevant costs and total interest earned and payable are same as the previous case 1 when $\alpha \in[0,0.5]$ but the values of $a_{L}(\alpha)$ and $a_{R}(\alpha)$ are different from the previous case 1 . In this case

$$
\begin{aligned}
& a_{L}(\alpha)=\frac{\left(r_{1}-\theta_{l} r_{2}\right)+\left(r_{2}-r_{1}-\theta_{l} r_{2}\right) \alpha}{\left(1-\theta_{l}\right)+2 \theta_{l} \alpha}, \\
& a_{R}(\alpha)=\frac{\left(r_{4}-\theta_{l} r_{3}\right)+\left(r_{4}-r_{3}-2 \theta_{l} r_{3}\right) \alpha}{\left(1-\theta_{l}\right)+2 \theta_{l} \alpha}
\end{aligned}
$$

Here the objective function for $\alpha \in[0.5,1]$ have the same expression as previous Case 1 where $\alpha \in[0,0.5]$ with different values of $a_{L}(\alpha)$ and $a_{R}(\alpha)$

In case 2: $\mathrm{gH}$-(ii) differentiability, the above system reduces to

$$
\begin{aligned}
& \frac{d q_{L}}{d t}= \begin{cases}-a_{L} p^{-\epsilon} & 0 \leq t \leq t_{1} \\
(\mu-1) a_{R} p^{-\epsilon} & t_{1} \leq t \leq t_{2} \\
(\mu-1) p^{-\epsilon}\left(a_{R}+b q_{R}\right) & t_{2} \leq t \leq t_{3} \\
-p^{-\epsilon}\left(a_{L}+b q_{L}\right) & t_{3} \leq t \leq T\end{cases} \\
& \frac{d q_{R}}{d t}= \begin{cases}-a_{R} p^{-\epsilon} & 0 \leq t \leq t_{1} \\
(\mu-1) a_{L} p^{-\epsilon} & t_{1} \leq t \leq t_{2} \\
(\mu-1) p^{-\epsilon}\left(a_{L}+b q_{L}\right) & t_{2} \leq t \leq t_{3} \\
-p^{-\epsilon}\left(a_{R}+b q_{R}\right) & t_{3} \leq t \leq T\end{cases}
\end{aligned}
$$

After solving using the boundary conditions, $q(0)=q\left(t_{2}\right)=q(T)=0$ and $q\left(t_{2}\right)=Q_{m}$, we get

$$
q_{L}(t)= \begin{cases}-a_{L} p^{-\epsilon} t & 0 \leq t \leq t_{1} \\ (\mu-1) a_{R} p^{-\epsilon}\left(t-t_{2}\right) & t_{1} \leq t \leq t_{2} \\ c_{1} e^{b k t}+c_{2} e^{-b k t}-\frac{a_{L}}{b} & t_{2} \leq t \leq t_{3} \\ k_{1} e^{r_{2} t}+k_{2} e^{-r_{2} t}+\frac{a_{L}}{b} & t_{3} \leq t \leq T\end{cases}
$$




$$
q_{R}(t)= \begin{cases}-a_{R} p^{-\epsilon} t & 0 \leq t \leq t_{1} \\ (\mu-1) a_{L} p^{-\epsilon}\left(t-t_{2}\right) & t_{1} \leq t \leq t_{2} \\ c_{1} e^{b k t}-c_{2} e^{-b k t}-\frac{a_{R}}{b} & t_{2} \leq t \leq t_{3} \\ -k_{1} e^{r_{2} t}+k_{2} e^{-r_{2} t}+\frac{a_{R}}{b} & t_{3} \leq t \leq T\end{cases}
$$

Where $k=(\mu-1) p^{-\epsilon} r_{2}=-b p^{-\epsilon}$

$$
\begin{aligned}
& c_{1}=\frac{1}{e^{2 b k t_{3}}-e^{2 b k t_{2}}}\left[\left(Q_{m}+\frac{a_{L}}{b}\right) e^{b k t_{3}}-\frac{a_{L}}{b} e^{b k t_{2}}\right] \\
& c_{2}=\frac{a_{L}}{b} e^{b k t_{2}}-\frac{1}{e^{2 b k\left(t_{3}-t_{2}\right)}}\left[\left(Q_{m}+\frac{a_{L}}{b}\right) e^{b k t_{3}}-\frac{a_{L}}{b} e^{b k t_{2}}\right] \\
& k_{1}=\frac{a_{R}-a_{L}}{2 b} e^{-c_{2} T} \\
& k_{2}=-\frac{a_{L}+a_{R}}{2 b} e^{c_{2} T} \\
& a_{L}(\alpha)=\frac{\left(r_{1}-\theta_{l} r_{2}\right)+\left(r_{2}-r_{1}-\theta_{l} r_{2}\right) \alpha}{\left(1-\theta_{l}\right)+2 \theta_{l} \alpha} \\
& a_{R}(\alpha)=\frac{\left(r_{4}-\theta_{l} r_{3}\right)+\left(r_{4}-r_{3}-2 \theta_{l} r_{3}\right) \alpha}{\left(1-\theta_{l}\right)+2 \theta_{l} \alpha}
\end{aligned}
$$

In this case also all the relevant costs and total interest earned and payable are same as the previous case 2 when $\alpha \in[0,0.5]$ but the values of $a_{L}(\alpha)$ and $a_{R}(\alpha)$ are different from the previous case 2 . In this case

$$
\begin{aligned}
& \mathrm{Z} a_{L}(\alpha)=\frac{\left(r_{1}-\theta_{l} r_{2}\right)+\left(r_{2}-r_{1}-\theta_{l} r_{2}\right) \alpha}{\left(1-\theta_{l}\right)+2 \theta_{l} \alpha}, \\
& a_{R}(\alpha)=\frac{\left(r_{4}-\theta_{l} r_{3}\right)+\left(r_{4}-r_{3}-2 \theta_{l} r_{3}\right) \alpha}{\left(1-\theta_{l}\right)+2 \theta_{l} \alpha}
\end{aligned}
$$

Here the objective function for $\alpha \in[0.5,1]$ have the same expression as previous Case 2 where $\alpha \in[0,0.5]$ with different values of $a_{L}(\alpha)$ and $a_{R}(\alpha)$

\section{Solution procedure for solving multi-objective non-linear problem}

The foregoing discussion provides a methodology for converting interval valued fuzzy differential equation into system of ordinary differential equation via generalized Hukuhara derivative approach. The $\alpha$-cut on the profit function and the emission function leads to a system of objective functions which have been solved by multi-objective genetic algorithm. As the developed problem arise so many parameters and handle this problem with classical methods will be very critical. Hence we applied the meta-heuristic multi-objective genetic algorithm method.

\section{Multi-objective genetic algorithm}

Genetic algorithm (GA) is a heuristic search algorithm used in computing to find true or approximate solutions in optimization which mimics the process of natural genetics i.e., survival of the fittest. It has five phases i.e., initial population, fitness function, selection, crossover, mutation. Parents are selected according to their fitness values. The better chromosomes have more chances to be selected. In this method, a few good chromosomes are used for creating new offspring in every iteration. Then some bad chromosomes are removed and the new offspring is placed in their places. The rest of population migrates to the next generation without going through the selection process. A multi-objective optimization problem involves a number of objective functions which are to be either minimized or maximized. As in a single-objective optimization problem, the multi-objective optimization problem may contain a number of constraints which have feasible solution (including all optimal solutions) to be satisfy. Since objectives can be either minimized or maximized, the multi-objective optimization problem in its general form can be written 
as

Minimize/Maximize $f(x)=\left\{f_{1}(x), f_{2}(x), f_{3}(x), \ldots \ldots, f_{k}(x)\right\}$

$x=\left\{x_{1}, x_{2}, x_{3}, \ldots . ., x_{z}\right\}$

subject to:

$g(x) \geq 0$

$h(x)=0$

$x^{l} \leq x \leq x^{u}$

Where $f$ is a vector comprising of $\mathrm{k}$ objective functions and $\mathrm{x}$ is a vector comprising of $\mathrm{z}$ solutions. $\mathrm{g}$ and $\mathrm{h}$ are vectors corresponding to inequality and equality constraints respectively. The lower bound and upper bound of the vector $\mathrm{x}$ is $x^{l}$ and $x^{u}$. The solutions of a multi-objective optimization problem are known as pareto optimal solutions.

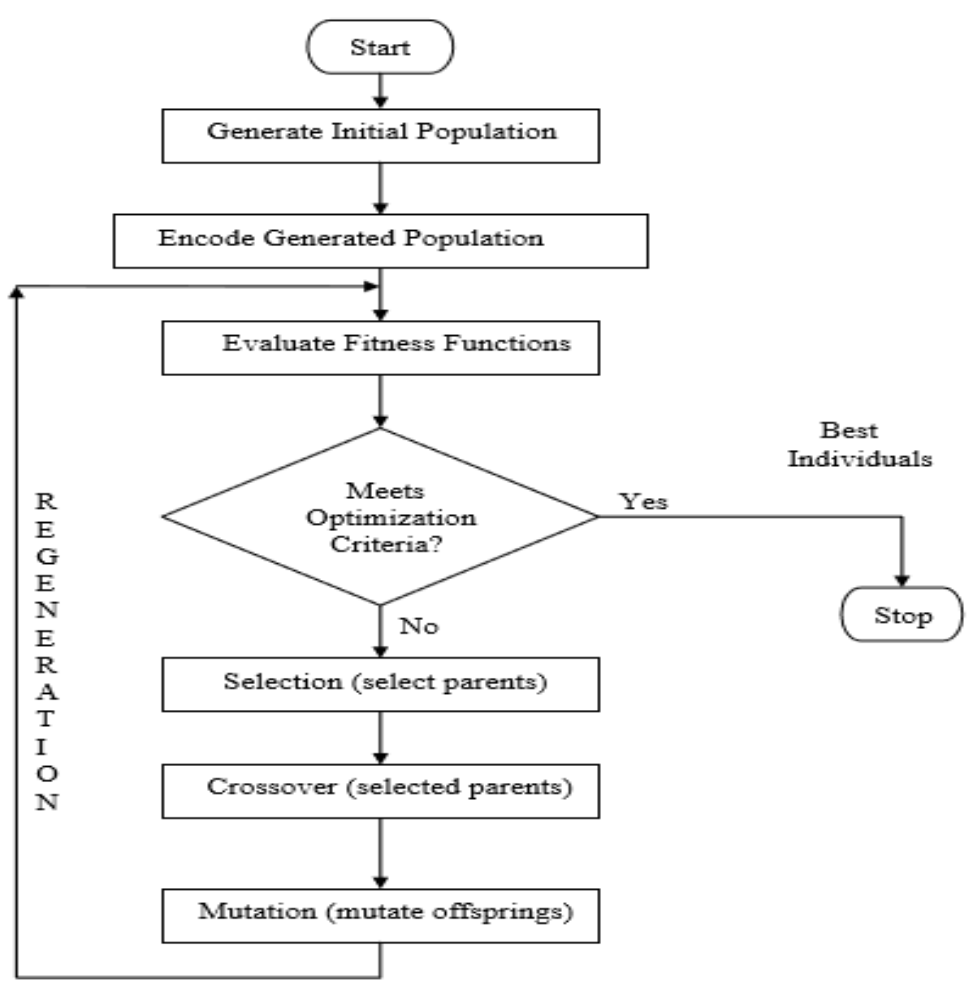

Figure 1. Graphical representation of procedure of GA

\section{Real life numerical data and estimation to type-2 fuzzy data}

"TATA Motors Limited" a well famous Indian multinational automotive manufacturing company manufactures passengers cars, trucks, vans, buses, sports car, construction equipment etc. The demands of these items from the suppliers are not fixed in every month. A group of managements decisions over the demand of these items are fuzzy in nature and the final decisions by chief production manager over the expert's decision is taken as type-2 fuzzy variable, more precisely trapezoidal type-2 fuzzy variable. Also, the company have to pay carbon emission cost due to the emission creates for obsolescence products, production units and to hold the manufacturing products. We have collected the data for January, 2017 and the corresponding input values in reduced and approximate form are given values. 
Let $C_{s}=45 \$$ unit, $\mu=1.8, \quad p=\$ 30 C_{h c}=7 \$ / m^{3}, \quad b^{\prime}=0.5 m^{3} /$ unit,$\alpha^{\prime}=$ $0.05, C_{s}^{\prime}=20 \$ /$ unit,$C_{3}=25 \$ /$ unit,$C_{o c}=5 \$ /$ ton $, i_{p}=0.61, \quad i_{e}=1.75, \alpha=$ 0.3 (when $\alpha \in[0,0.5]$ ), $\alpha=0.7$ (when $\alpha \in[0.5,1]$ ), $\quad b=0.8 \quad \epsilon=0.7 \quad C_{m c}=3 \$$ /unit, $\quad C_{h}=$ $6 \$ / m^{3}, \quad C_{b}=1.02 \$ / m^{3} \quad a^{\prime}=0.8$ ton/unit, $\quad\left(r_{1}, r_{2}, r_{3}, r_{4}, \theta_{l}, \theta_{r}\right)=(10,12,14,16,0.5,0.3)$

Table-2: Optimization results for different sub cases for type-2 fuzzy demand by using Multi-objective Genetic algorithm

\begin{tabular}{|c|c|c|c|c|c|c|c|}
\hline \multicolumn{2}{|c|}{ Different cases } & sub cases & $T$ & $M$ & $N$ & {$\left[T P_{L}, T P_{R}\right]$} & {$\left[T E_{L}, T E_{R}\right]$} \\
\hline$\alpha \in[0,0.5]$ & Case 1 & 1.1 & 1.2567 & 0.8257 & 0.6758 & {$[213.91,368.08]$} & {$[106.82,171.12]$} \\
\cline { 3 - 7 } & 1.2 & 1.3459 & 0.9256 & 0.7584 & {$[433.67,597.31]$} & {$[213.56,297.65]$} \\
\cline { 3 - 8 } & 1.3 & 1.1346 & 1.5682 & 0.8496 & {$[609.68,779.84]$} & {$[305.57,387.69]$} \\
\cline { 3 - 8 } & \multirow{3}{*}{ Case 2 } & 2.1 & 1.1438 & 0.8391 & 0.7143 & {$[232.58,378.37]$} & {$[118.64,185.97]$} \\
\cline { 3 - 7 } & & 2.2 & 1.2337 & 0.9123 & 0.7852 & {$[445.72,605.38]$} & {$[221.37,307.82]$} \\
\cline { 3 - 7 } & 2.3 & 1.3258 & 1.7235 & 1.1324 & {$[618.67,796.57]$} & {$[312.67,396.78]$} \\
\hline \multirow{2}{*}{ Case 1 } & 1.1 & 1.3457 & 0.8472 & 0.7523 & {$[198.78,275.86]$} & {$[118.23,185.79]$} \\
\cline { 3 - 7 } & 1.2 & 1.5676 & 0.8472 & 0.7523 & {$[412.37,576.28]$} & {$[227.52,307.64]$} \\
\cline { 3 - 7 } & & 1.3 & 1.1256 & 1.4328 & 0.9726 & {$[582.57,680.19]$} & {$[331.24,415.28]$} \\
\cline { 3 - 7 } & Case 2 & 2.1 & 1.058 & 0.7581 & 0.7058 & {$[223.81,353.57]$} & {$[127.64,196.67]$} \\
\cline { 3 - 7 } & 2.2 & 1.123 & 0.7638 & 0.7253 & {$[432.35,575.83]$} & {$[231.58,316.97]$} \\
\cline { 3 - 7 } & & 2.3 & 1.321 & 1.873 & 1.0357 & {$[608.67,721.64]$} & {$[327.67,409.61]$} \\
\hline
\end{tabular}

Table-3: Effects of unit selling price $C_{s}$ on profit function via gH-(i) differentiability of different sub cases of Case 1 for type-2 fuzzy demand when $\alpha \in(0,0.5]$

\begin{tabular}{|c|c|c|c|}
\hline \multirow{2}{*}{$C_{s}(\$)$} & Sub-case 1.1 & Sub-case 1.2 & Sub-case 1.3 \\
\cline { 2 - 4 } & {$\left[T P_{L}, T P_{R}\right]$} & {$\left[T P_{L}, T P_{R}\right]$} & {$\left[T P_{L}, T P_{R}\right]$} \\
\hline 45 & {$[213.91,368.08]$} & {$[433.67,597.31]$} & {$[609.68,779.84]$} \\
\hline 47 & {$[237.81,381.15]$} & {$[457.28,605.35]$} & {$[602.13,797.23]$} \\
\hline 49 & {$[251.26,398.57]$} & {$[478.63,621.46]$} & {$[641.25,809.15]$} \\
\hline 51 & {$[273.43,418.93]$} & {$[493.54,639.56]$} & {$[657.81,822.21]$} \\
\hline 53 & {$[287.19,429.35]$} & {$[506.23,652.21]$} & {$[662.23,832.32]$} \\
\hline 55 & {$[293.25,444.61]$} & {$[517.41,667.82]$} & {$[671.82,843.67]$} \\
\hline 57 & {$[302.61,459.82]$} & {$[529.35,679.81]$} & {$[682.56,857.67]$} \\
\hline
\end{tabular}

Table-4: Effects of unit purchasing cost $p$ on profit function via gH-(ii) differentiabi- lity of different sub cases of Case 2 for type-2 fuzzy demand when $\alpha \in(0,0.5]$

\begin{tabular}{|c|c|c|c|}
\hline \multirow{2}{*}{$p(\$)$} & Sub-case 2.1 & Sub-case 2.2 & Sub-case 2.3 \\
\cline { 2 - 4 } & {$\left[T P_{L}, T P_{R}\right]$} & {$\left[T P_{L}, T P_{R}\right]$} & {$\left[T P_{L}, T P_{R}\right]$} \\
\hline 30 & {$[232.58,378.37]$} & {$[445.72,605.38]$} & {$[618.67,796.57]$} \\
\hline 32 & {$[227.64,369.87]$} & {$[438.79,591.25]$} & {$[611.52,787.23]$} \\
\hline 34 & {$[221.23,362.51]$} & {$[432.81,583.71]$} & {$[603.15,779.14]$} \\
\hline 36 & {$[218.21,356.10]$} & {$[426.75,575.23]$} & {$[596.45,769.23]$} \\
\hline 38 & {$[211.37,349.58]$} & {$[420.12,562.14]$} & {$[589.64,761.42]$} \\
\hline 40 & {$[202.51,341.78]$} & {$[413.25,557.69]$} & {$[581.23,756.21]$} \\
\hline 42 & {$[196.25,335.62]$} & {$[402.72,551.13]$} & {$[571.51,749.17]$} \\
\hline
\end{tabular}


Table-5: Effects of unit obsolescence rate $\alpha^{\prime}$ on emission function via gH-(i) differentiability of different sub cases of Case 1 for type-2 fuzzy demand when $\alpha \in(0.5,1]$

\begin{tabular}{|c|c|c|c|}
\hline \multirow{2}{*}{$\alpha^{\prime}$} & Sub-case 1.1 & Sub-case 1.2 & Sub-case 1.3 \\
\cline { 2 - 4 } & {$\left[T E_{L}, T E_{R}\right]$} & {$\left[T E_{L}, T E_{R}\right]$} & {$\left[T E_{L}, T E_{R}\right]$} \\
\hline 0.05 & {$[118.23,185.97]$} & {$[227.52,307.64]$} & {$[331.24,415.28]$} \\
\hline 0.10 & {$[123.19,192.25]$} & {$[233.67,315.19]$} & {$[338.75,423.18]$} \\
\hline 0.15 & {$[129.20,198.75]$} & {$[237.15,322.50]$} & {$[343.16,429.11]$} \\
\hline 0.20 & {$[136.49,207.26]$} & {$[242.62,329.23]$} & {$[349.21,436.07]$} \\
\hline 0.25 & {$[142.15,211.27]$} & {$[249.13,337.19]$} & {$[356.16,442.18]$} \\
\hline 0.30 & {$[149.35,220.05]$} & {$[256.27,342.56]$} & {$[361.23,451.95]$} \\
\hline 0.35 & {$[156.07,227.18]$} & {$[261.81,347.25]$} & {$[369.09,459.67]$} \\
\hline
\end{tabular}

Table-6: Effects of weight of obsolescence product $a^{\prime}$ on emission function via gH-(ii) differentiability of different sub cases of Case 2 for type-2 fuzzy demand when $\alpha \in(0.5,1]$

\begin{tabular}{|c|c|c|c|}
\hline$a^{\prime}$ & Sub-case 2.1 & Sub-case 2.2 & Sub-case 2.3 \\
\cline { 2 - 4 } & {$\left[T E_{L}, T E_{R}\right]$} & {$\left[T E_{L}, T E_{R}\right]$} & {$\left[T E_{L}, T E_{R}\right]$} \\
\hline 0.8 & {$[127.64,196.67]$} & {$[231.58,316.97]$} & {$[327.67,409.61]$} \\
\hline 1.0 & {$[131.25,201.25]$} & {$[237.05,321.84]$} & {$[334.25,416.87]$} \\
\hline 1.2 & {$[136.71,212.08]$} & {$[239.17,329.15]$} & {$[339.51,421.75]$} \\
\hline 1.4 & {$[141.81,216.25]$} & {$[244.23,336.91]$} & {$[343.61,429.82]$} \\
\hline 1.6 & {$[146.34,218.31]$} & {$[249.71,341.28]$} & {$[349.71,437.71]$} \\
\hline 1.8 & {$[153.81,225.83]$} & {$[255.82,346.17]$} & {$[356.82,442.82]$} \\
\hline 2.0 & {$[157.35,231.19]$} & {$[261.09,351.25]$} & {$[360.08,446.72]$} \\
\hline
\end{tabular}

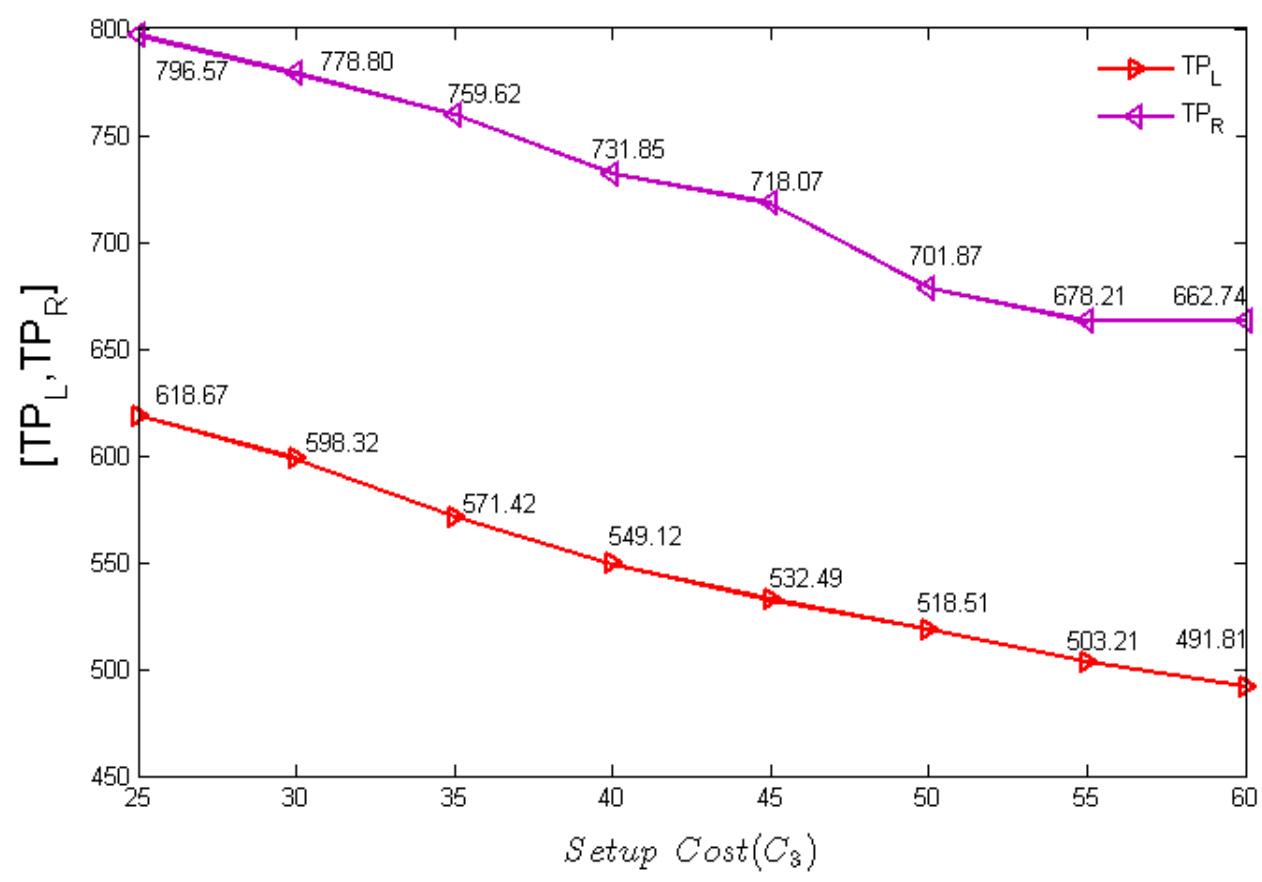

Figure 2. Effect of set-up cost on profit function for sub-case 2.3 when $\alpha \in[0,0.5]$ 


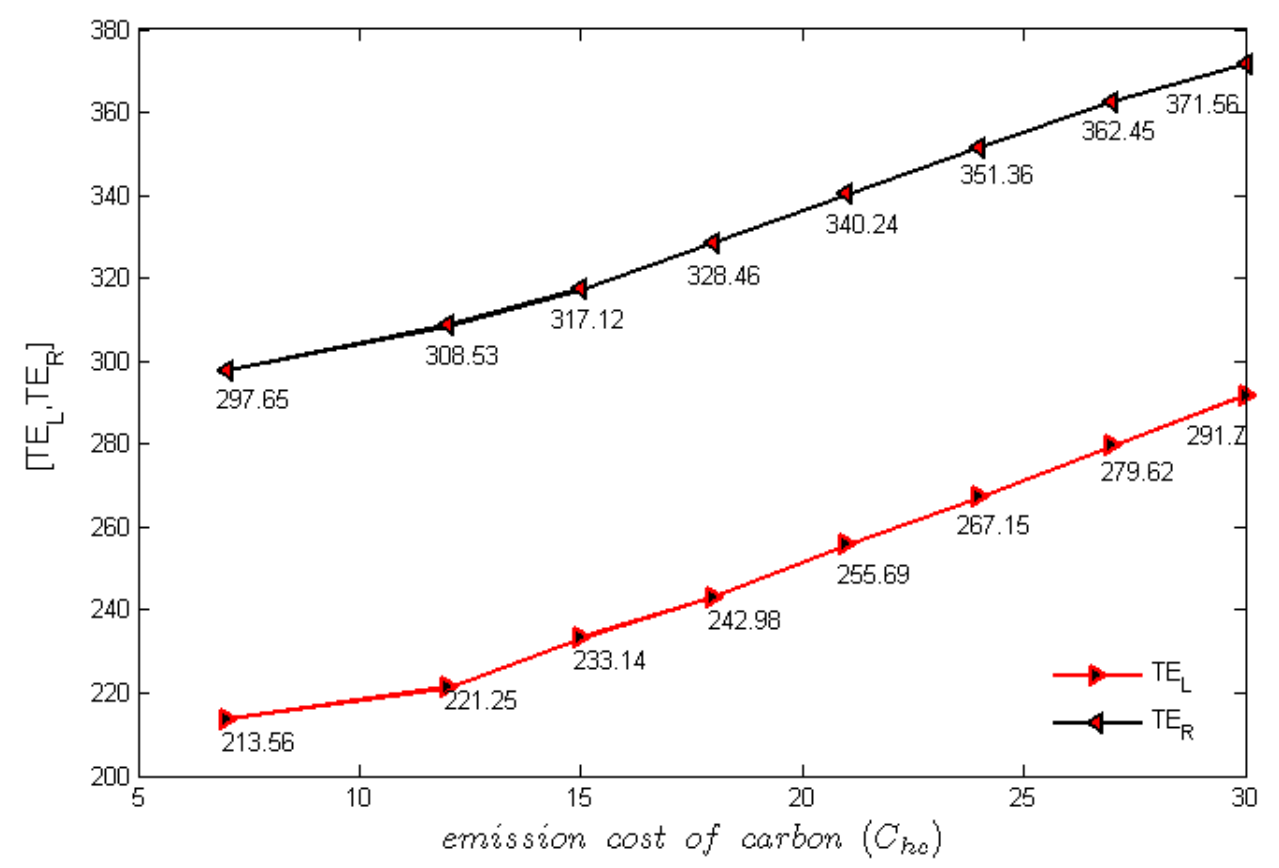

Figure 3. Effect of emission cost of carbon on emission function for sub-case 1.1 when $\alpha \in[0,0.5]$

\section{Discussion}

Table 2 describes the optimal results for the profit function and the emission function in interval form and concluded that when the credit period of retailer is greater than total cycle time $\mathrm{T}$ via $\mathrm{gH}$-(i) differentiability, the profit is maximum, i.e. [609.68, 779.84] and minimum emission is calculated as $[106.82,171.12]$ for sub-case 1.1 when $\alpha \in[0,0.5]$. When the profit and emission is calculated via gH-(ii) differentiability, we also observe that as the credit period of retailer is greater than total cycle time $\mathrm{T}$, the profit is maximum, i.e. [618.67, 796.57] and minimum emission is calculated as [118.64, 185.97] for sub-case 2.1 when $\alpha \in[0,0.5]$. As one can easily observed from Table 2 that the same scenario is depicted for $\alpha \in[0.5,1]$. In this case sub-case 2.3 gives the maximum profit, i.e. [608.67, 721.64]. We can also conclude that in case of sub-case 1.1, the emission cost is minimum, i.e. $[106.82,171.12]$ when $\alpha \in[0,0.5]$. We observe the effect of unit selling price $\left(C_{s}\right)$ on profit function and can conclude that with the increase of unit selling price, the profit function is also increasing as depicted in Table 3. Table 4 analyses the effect of unit purchasing cost $(p)$ on profit function via gH-(ii) differentiability when $\alpha \in[0,0.5]$ and observe that with the increase of unit purchasing cost the total profit of each sub-case is decrease. Table 5 shows that if the unit obsolescence rate $\alpha^{\prime}$ is increase for type 2 fuzzy demand over time for $\alpha \in[0.5,1]$ corresponding cost of emission is also increase. From Table 6 it observed that with the increasing values of weight of obsolescence product $a^{\prime}$, total emission cost for each sub-cases are also increased. With the increase of set-up cost, the profit function is decreasing as depicted in Figure 2 when $\alpha \in[0,0.5]$. We can observe from Figure 3 that the total emission cost is increasing as the emission cost of carbon is increasing.

\section{Conclusions and future research work:}

The present analyses of the model specifically introduce the concept of type- 2 fuzzy variable can be taken as a key factor for a decision maker (DM) engaged with the demand 
unit. Demand of an item in market is always fluctuating and in this present model this fluctuation is measured by trapezoidal type-2 fuzzy variable. The present model illustrated a new direction in the field of inventory modeling applying the adventure of Mathematics. The DM is able to take more appropriate precise decisions with the help of present analyses.

In this paper, some useful ideas are presented to deal with inventory control problem with type-2 fuzzy parameters. Along with the main contributions discussed in introduction some more aspects are as follows.

1. CV based reduction method proposed by Qin et al. [23] is discussed and successfully applied to the proposed model to find the total profit function and emission function.

2. According to literature survey for the first time in a single mathematical formulation, we introduced an economic production quantity model with demand depends on the production price and stock in fuzzy environment where demand is taken as trapezoidal type-2 fuzzy number. With the use of CV based reduction method and $\alpha$-cut of hexagonal fuzzy number the proposed model is solved to find maximum profit and minimum cost of emission of carbon.

3. Some new real life based important facts are provided and discussed in this paper, which will help in developing the business management.

As a future work the presented models can be extended to different types of inventory problems including price discounts, quantity discounts, taking selling price, ordering cost as triangular fuzzy number, intuitonistic number, triangular type-2 fuzzy number, gamma type-2 fuzzy number, Gaussian type-2 fuzzy number etc .

As it is assumed that the unit selling price is greater than the unit purchasing price, the retailer must have sufficient amounts before the end of business period and to pay the dues to the wholesaler some time before the end of the total cycle and in this situation, he will have to pay less interest to the wholesaler. Moreover, the retailer can earn more interest after that time up to the end of the business period. This new approach to calculate the interest earned by the retailer may also apply in this model and the result can be compared with the conventional approach also. The concept of immediate part payment and the delay-payment for the rest can also allowed by the wholesaler for an item over a finite planning horizon or random planning horizon In addition, against an immediate part payment (variable) to the wholesaler, there is a provision for (i) borrowing money from a money lending source and (ii) earning some relaxation on credit period from the wholesaler. The models can also be developed with respect to the retailer for maximum profit. We can also extend the current model for partial trade credit i.e. supplier offers partial trade credit to retailer and retailer offers full trade credit to customers.

\section{References}

[1] S.M. Aljazzar, A. Gurtu and M.Y. Jaber, Delay in payments- A strategy to reduce carbon emissions from supply chains, J. Clean. Prod. 170, 636-644, 2018.

[2] T. Allahviranloo and M. Afsher Kermani, Numerical methods for fuzzy linear partial differential equations under new definition for derivative, Iran. J. Fuzzy Syst. 7 (3), 33-50, 2010.

[3] D. Battini, A. Persona and F. Sgarbossa, A Sustainable EOQ model: Theoretical formulation and applications, Int. J. Prod. Econ. 149, 145-153, 2014.

[4] B. Bede, A note on two-point boundary value problems associated with non-linear fuzzy differential equations, Fuzzy Sets and Systems 157, 986-989, 2006.

[5] B. Bede and S.G. Gal, Generalizations of the differentiability of fuzzy-number-valued functions with applications to fuzzy differential equations, Fuzzy Sets and Systems 151, 581-599, 2005.

[6] S.C. Chang, Fuzzy production inventory for fuzzy product quantity with triangular fuzzy number, Fuzzy Sets and Systems 107, 37-57, 1999. 
[7] Y. Chen and L. Zhang, Some new results about arithmetic of type-2 fuzzy variables, Journal of Uncertain System 5 (3), 227-240, 2011.

[8] D. Dubois and H. Prade, Operations on fuzzy numbers, Int. J. Syst. Sci. 9 (6), 613-626, 1978.

[9] R. Ezzati, K. Maleknejad and S. Khezertoo, Convergence, Consistency and stability in fuzzy differential equations, Iran. J. Fuzzy Syst. 12 (3), 95-112, 2015.

[10] P. Guchhait, M.K. Maiti and M. Maiti, A production inventory model with fuzzy production and demand using fuzzy differential equation: An interval compared genetic algorithm approach, Eng. Appl. Artif. Intell. 26, 766-778, 2013.

[11] V. Hovelaque and L. Bironneau, The carbon-constrained EOQ model with carbon emission dependent demand, Int. J. Prod. Econ. 164, 285-291, 2015.

[12] M. Jonas, M. Marland, W. Winiwarter, T. White, Z. Nahorski, R. Bun and S. Nilsson, Benefits of dealing with uncertainty in greenhouse gas inventories: introduction, Climatic Change 103 (1-2), 175-213, 2010b.

[13] A. Kandel and W.J. Byatt, Fuzzy differential equations, In Proceedings of the International Conference on Cybernetics and Society, Tokyo, 1213-1216, 1978.

[14] N. Kazemi, S.H. Abdul-Rashid, R.A.R. Ghazila, E. Shekarian and S. Zanoni, Economic order quantity models for items with imperfect quality and emission considerations, Int. J. Syst. Sci.: Oper. \& Logist. 5 (2), 99-115, 2018.

[15] P. Kundu, S. Kar and M. Maiti, Fixed charge transportation problem with type-2 fuzzy variables, Inf. Sci. 255, 170-184, 2014.

[16] P. Kundu, S. Kar and M. Maiti, Multi-item solid transportation problem with type-2 fuzzy parameters, Appl. Soft Comput. 31, 61-80, 2015.

[17] H.M. Lee and J.S. Yao, Economic production quantity for fuzzy demand quantity and fuzzy production quantity, European J. Oper. Res. 109, 203-211, 1998.

[18] D.C. Lin and J.S. Yao, Fuzzy economic production for production inventory, Fuzzy Sets and Systems 111, 465-495, 2000.

[19] Z.Q. Liu and Y.K. Liu, Type-2 fuzzy variables and their arithmetic, Soft Computing 14, 729-747, 2010.

[20] P. Majumder, S.P. Mondal, U.K. Bera and M. Maiti, Application of Generalized Hukuhara derivative approach in an economic production quantity model with partial trade credit policy under fuzzy environment, Operations Research Perspective 3, 7791, 2016.

[21] S. Miller, M. Gongora and R. John, Interval type-2 fuzzy modeling and simulated annealing for real world inventory management, International Conference on Hybrid Artificial Intelligence Systems, 231-238, Springer-Verlag Bertin, Heidelberg, 2011.

[22] M. Mizumoto and K. Tanaka, Fuzzy sets of type-2 under algebraic product and algebraic sum, Fuzzy Sets and Systems 5 (3), 277-280, 1981.

[23] R. Qin, Y.K. Liu and Z.Q. Liu, Methods of critical value reduction for type-2 fuzzy variables and their applications, J. Comput. Appl. Math. 235, 1454-1481, 2011.

[24] P. Rajarajeswari, A.S. Sudha and R. Karthika, A New Operation on Hexagonal Fuzzy Number, Int. J. Fuzzy Log. Syst. 3 (3), 15-26, 2013.

[25] J. Sadeghi, S.T.A. Niaki, M. Malekian and Y. Wang, A Lagrangian relaxation for a fuzzy random EPQ problem with shortages and redundancy allocation: two tuned meta-heuristics, Int. J. Fuzzy Syst. 20 (2), 515-533, 2018.

[26] S. Sharan, S.P. Tiwary and V.K. Yadav, Interval type-2 fuzzy rough sets and interval type-2 fuzzy closure spaces, Iran. J. Fuzzy Syst. 12 (3), 113-125, 2015.

[27] E. Shekarian, C.H. Glock, S.M.P. Amiri and K. Schwindl, Optimal manufacturing lot size for a single-stage production system with rework in a fuzzy environment, J. Intell. Fuzzy Syst. 27 (6), 3067-3080, 2014. 
[28] E. Shekarian, M.Y. Jaber, N. Kazemi and E. Ehsani, A fuzzified version of the economic production quantity (EPQ) model with backorders and rework for a single stage system, Eur. J. Ind. Eng. 8 (3), 291-324, 2014.

[29] E. Shekarian, N. Kazemi, S.H. Abdul-Rashid and E.U. Olugu, Fuzzy inventory models: A comprehensive review, Appl. Soft Comput. 55, 588-621, 2017.

[30] E. Shekarian, E.U. Olugu, S.H. Abdul-Rashid and E. Bottani, A fuzzy reverse logistics inventory system integrating economic order/production quantity models, Int. J. Fuzzy Syst. 18 (6), 1141-1161, 2016.

[31] E. Shekarian, E.U. Olugu, S.H. Abdul-Rashid and N. Kazemi, An economic order quantity model considering different holding costs for imperfect quality items subject to fuzziness and learning, J. Intell. Fuzzy Syst. 30 (5), 1985-2997, 2016.

[32] H.N. Soni, B. Sarkar and M. Joshi, Demand uncertainty and learning in fuzziness in a continuous review inventory model, J. Intell. Fuzzy Syst. 33 (4), 2595-2608, 2017.

[33] L. Stefanini and B. Bede, Generalized Hukuhara differentiability of interval-valued functions and interval differential equations, Nonlinear Anal. 71, 1311-1328, 2009.

[34] J.R. Stock, S.L. Boyer and T. Harmon, Research opportunities in supply chain management, J. Acad. Mark. Sci. 38 (1), 32-41, 2010.

[35] Z. Takac, Inclusion and subsethood measure for interval-valued fuzzy sets and for continuous type-2 fuzzy sets, Fuzzy Sets and Systems 224, 106-120, 2013.

[36] E.J. Villamizar-Roa, V. Angulo-Castilo and Y. Chaleo-Cano, Existence of solutions to fuzzy differential equation with generalized Hukuhara derivative via contractive-like mapping principles, Fuzzy Sets and Systems 265, 24-38, 2015.

[37] H.C. Wu, The central limit theorems for fuzzy random variables, Inf. Sci. 120, 239256, 1999.

[38] L.A. Zadeh, The concept of a linguistic variable and its application to approximate reasoning - I, Inf. Sci. 8, 199-249, 1975.

[39] L.A. Zadeh, The concept of a linguistic variable and its application to approximate reasoning - II, Inf. Sci. 8, 301-357, 1975. 